\title{
Enhancing Managerial Skills for Intern-Nurses by Using Problem-Based Learning Strategy
}

\author{
1 Asmaa Khaled Abd El-Aziz Zaki, 2Gehan Mohamed Ahmed Mostafa, 3Ebtesam Saeed \\ Ahmed
}

1 Assistant lecturer of Nursing Administration, Faculty of Nursing, Benha University

2 Professor of Nursing Administration, Faculty of Nursing, Helwan University.

3 Assistant Professor of Nursing Administration, Faculty of Nursing, Benha University

\begin{abstract}
Background: Problem-based learning (PBL) has been implemented throughout the world as an innovative learning method in nursing education and some Egyptian universities have adopted PBL as a learning method to enhance intern-nurses application of knowledge, promotes self-learning, and develops managerial skills. The study aimed to enhance managerial skills for intern-nurses by using problem-based learning strategy. Study Design: A quasi-experimental design was utilized. The study sample: A convenient sample of 124 intern-nurses divided into two groups; Traditional based learning (TBL) group (70 intern-nurses) and PBL group (54 intern-nurses). Study Setting: The study was conducted in the intern-nurses' clinical training areas at Benha University Hospital. Tools: Five tools were used for data collection; (1) Intern-nurses' opinionnaire sheet, (2) Managerial skills knowledge questionnaire, (3) Managerial skills' practice questionnaire, (4) Attitude toward PBL questionnaire and (5) Evaluation questionnaire. Results: The majority of intern-nurses $(92.6 \%$ \& $72.2 \%$ ) preferred PBL at immediate post and follow up phases respectively than TBL as a strategy of learning. Also, the majority of intern-nurses $(96.3 \%$ \& $87.1 \%)$ had satisfactory level of managerial skills knowledge at PBL and TBL group respectively during immediate post program phase. The majority of intern-nurses $(87.0 \%)$ had positive attitude toward PBL at follow up phase compared to pre-program. Conclusion: There was highly statistically significant difference improvement found in the immediate post program between both groups with higher total mean scores in PBL group than TBL group during immediate post program. Also, there was highly statistically significant positive correlation between total managerial skills' knowledge and total managerial skills' practice at both groups. Recommendations: The study recommended that PBL strategy should be adopted by Faculty of Nursing Benha University for better skills and learning outcome, to allow students' interactions and encourage higher thinking level.
\end{abstract}

Key words: Intern-nurses, Managerial skills, Problem-based learning (PBL). 


\section{Asmaa Khaled Abd El-Aziz, Gehan Mohamed Ahmed Mostafa, Ebtesam Saeed Ahmed}

\section{Introduction}

Intern-nurses are baccalaureate nursing graduates enrolled in the internship year to gain practice in a real world setting under the guidance of knowledgeable, experienced, and successful supervisor. The nursing internship year is the final clinical year, where intern-nurses are exposed to the direct clinical experience of patient care in a healthcare setting and is part of the degree requirements. Clinical experiences include the rotation of intern-nurses through various clinical setting for 12 months (Bakr et al., 2013 and AlThiga et al., 2017).

The internship training course is related to different specialty of nursing such as critical care unit, dialysis unit, cardiac catheterization, medical-surgical specialties, cardiothoracic surgery, maternal and newborn health nursing, pediatric unit and nursing administration where intern-nurses are trained clinically through these specialties (Makhlof and El-Saman, 2017). The nursing discipline is growing, and higher education in nursing aims to prepare students for their future work beyond the classroom and to develop their skills to become independent professionals (Othman and shalaby, 2014; Mahmoud, 2017; Rakhudu et al., 2017).

Professional nurses must be able to exercise their multiple roles of care providers, managers, teachers, as well as lifelong learners and researchers. To do so, they should be able to solve problems through analysis, synthesis, critical thinking, effective communication and others skills (El-Sayed et al., 2013). Managerial skills are parts of managerial competencies. Management skills identify abilities or behaviors that are crucial to success in managerial positions. The term "skill" refers to the ability to do something in an effective manner. Managerial skills are defined as a manager's ability to transform information and knowledge into practice (Bulog et al., 2017).

Three areas of skills needed by managers in organizations included: firstly, technical skill; the ability and efficiency of a particular type of activities that is consistent with the methods, procedures and techniques related to the activity. This skill required specific information, analytical talent and expertise to create and use of the working tools. Secondly, human relation skill; the ability of managers to work effectively as a member of the group and making collective efforts within the group. Finally, conceptual skill; manager's ability to observe the whole picture of the organization and how the different parts work together (Esfandnia et al., 2016).

The Nursing Education Society in Egypt faces a challenge in preparing students to execute nursing roles within the context of integrated health care system (Abd El-Raouf and Ahmed, 2011; Molande et al., 2017; Soliman et al., 2017). As changes in nursing require from nurses, autonomy and the ability to take relevant decisions, therefore, is one of the goals of nursing education to reduce the gap between theory and practice (Gönc et al., 2017). One of the proposed ways to eliminate the gap between academic and clinical performances is changing the traditional educational system to problem-based learning (PBL) (deNevers, 2014; Sayyah et al., 2017).

Problem-based learning is a studentcentered, problem-based, and small-group method of learning that emphasizes use of realistic scenarios. PBL places the responsibility for learning in the hands of the students, and is concerned with both what 
Enhancing Managerial Skills for Intern-Nurses by Using Problem-Based Learning Strategy

students learn and how they learn it (Adiga and Adiga, 2015; Keshk et al., 2016; Wang et al., 2016; Kartikeyan and Malgaonkar, 2018; Fatima et al., 2019; Kim, 2019). Moreover, problem-based learning is defined as the learning that results from the process of working toward the understanding or resolution of a problem under the guidance of a faculty named tutor (Ahamad et al., 2017; Wosinski et al., 2018). The tutor's role is to facilitate and guide students towards discovering answers independently instead of being given the answers, offering students a richer and more holistic learning experience (Othman et al., 2013; Heaviside et al., 2018).

Problem-based learning is an educational strategy and a method to organize the learning process in such a manner that the students are actively engaged in finding answers by themselves. The four major goals of PBL are; acquisition of the knowledge base underlying managerial practice, development of managerial skills, development of problemsolving skills, and development of lifelong learning skills (Mohamad, 2017).

Problem-based learning is a powerful learning approach offering many benefits including increased acquisition and retention of knowledge, stimulation of problem solving, enhancement of intrinsic subject interest, deep learning, enhanced communication, teamwork, presentation and critical appraisal skills, and enhanced clinical skills (Burgess et al., 2018; Kim, 2019). PBL have been proved to be a success in the enhancement and development of critical thinking ability, leadership qualities, self-directed learning, professional and interpersonal skills, management skills, collaborative learning, practicing empathy and many more (Ansari et al., 2015).

\section{Significance of the study}

Nursing education consists of both classroom and clinical experience. So, one of the important goal in nursing education is to promote the transfer of theory to practice. Also, knowledge in health care continues to expand and new technology develops, so, the demand for intern-nurses to be able to solve complex problems efficiently has also increased (Shin and Kim, 2013). Problembased learning is now extensively utilized within contemporary nursing education to assist intern-nurses to integrate nursing theory into practice. It promotes critical thinking, self-directed study and problem solving by using scenarios related to certain health conditions (Yang and Yang, 2013).

From the researcher's clinical experience, it was noticed that intern-nurses' knowledge retention was decreased, and in transitional stage from academic education to clinical experiences with more responsibilities and they are exposed for a threatening, difficult and different situations which internnurses couldn't deal and solve it. Also, internnurses' managerial skills and their situation handling were unsatisfactory. So, this study aimed to enhance managerial skills for internnurses by using problem-based learning strategy at Benha University Hospital.

\section{Aim of the Study}

The aim of this study was to:

Enhance managerial skills for internnurses by using problem based learning strategy at Benha University Hospital through:

1. Determining intern-nurses' opinion regarding traditional methods or problem-based learning.

2. Assessing intern-nurses' knowledge and skills regarding managerial skills. 


\section{Asmaa Khaled Abd El-Aziz, Gehan Mohamed Ahmed Mostafa, Ebtesam Saeed Ahmed}

3. Identifying intern-nurses' attitude toward problem-based learning.

4. Designing and implementing managerial skills program for internnurses by using problem-based learning strategy.

5. Evaluating the effectiveness of the designed program on intern-nurses' managerial knowledge and skills.

\section{Research hypothesis}

Applying problem-based learning strategy will significantly improve intern- nurses' managerial skills.

\section{Subjects and Method}

The methodology of the current study were portrayed under the following four designs;
I. Technical design
II. Administrative design
III. Operational design
IV. Statistical design

\section{Technical design}

The technical design of the current study included study design, study setting, subjects as well as tools of data collection.

\section{Study design:}

A quasi-experimental design was utilized to achieve the aim of the present study.

\section{Study setting:}

The current study was conducted in the intern-nurses' clinical training areas at Benha University Hospital according to the policy of internship as following:

1) In the basic areas: Medical-surgical units, intensive care units, pediatric units, maternal and newborn health nursing and nursing administration duty.

2) In the selective (optional) areas: Hemodialysis, cardiac catheterization unit, cardiothoracic surgery, brain and neuro-departments.

This study was conducted at Benha University Hospital, the total number of beds at this hospital is 880 . The hospital composed of three separated buildings; Medical building 478 beds, surgical building 384 beds and ophthalmology building 18 beds. There were nine critical care units; Intensive care unit (ICU), medium ICU, emergency ICU, hepatic ICU, cardiac care unit (CCU), chest ICU, chest and heart ICU, pediatric ICU and pediatric incubator ICU.

\section{Subjects:}

The study subject was consisted of a convenient sample of 124 intern-nurses who enrolled in the internship year 2018/ 2019 at the Faculty of Nursing. Their total number was 294 but 170 intern-nurses were excluded from the study for many reasons as; 133 of them transferred to other hospitals to complete their internship year, 3 postponed their internship year, and 34 intern-nurses refused to participate. So, the actual internnurses who participated in this study composed of 124 intern-nurses; 54 internnurses were placed in experimental group (study group) and the other 70 were placed in control group. The control group received traditional teaching methods (lecture) and the experimental group had their lessons based on problem-based learning (PBL) approach.

\section{Tools of data collection:}

Data of the present study was collected by using five tools as following;

Tool (I): Intern-nurses' opinionnaire sheet: A Structured questionnaire developed by the researcher based on reviewing the related literature review (Ghimire and Bhandary, 
2014; Ibrahim et al., 2014 and Majagi and Torgal, 2015). It consisted of two parts:-

Part (1): Personal characteristics of the studied intern-nurses as; age, gender, marital status, qualification before attachment to the faculty, previous working experience and years of work experience (6 questions).

Part (2): Consisted of 27 items to identify intern-nurses' opinion regarding traditional learning or PBL e.g. use previous relevant knowledge and experience, promote the retention of knowledge.

\section{Scoring system:}

The studied intern-nurses' responses were rated by using a three point Likert scale ranged from (1-3) as; agree (3 points), neutral (2 points) and disagree (1 point). Range of scores from (27-81). Each intern-nurse had chosen one best answer after reading carefully and understanding. Finally, the answer was calculated numerical values. Accordingly level of intern-nurses' preferences was categorized as the following; preferred $\geq 75 \%$ that equal $\geq$ 61 points and not preferred $<75 \%$ that equal $<$ 61 points (Ahmed, 2013).

\section{Tool (II): Managerial skills knowledge} questionnaire: A structured questionnaire developed by the researcher based on reviewing the related literature review (Sullivan and Decker, 2009; Kelly, 2010; Rubenfeld and Scheffer, 2010; Parsons, 2015 and Pryby, 2016) to assess internnurses' (experimental and control group) knowledge regarding conceptual (decision making), human relation (teamwork and conflict management) and technical skills (time management). It consisted of 25 questions divided into (15) multiple choice questions, (5) matching and (5) true or false questions.

\section{Scoring system:}

The studied intern-nurses' answers were compared with a model key answer and scored as; "2" for correct and "1" for incorrect. Range of scores from (25-50), in this respect the level of intern-nurses' knowledge was categorized as the following; Satisfactory level $\geq 60 \%$ equal to $\geq 30$ points and Unsatisfactory level< $60 \%$ equal to $<30$ points (Tayyeb, 2013 and Abd-Elmonem et al., 2019).

\section{Tool (III): Managerial skills' practice} questionnaire: It included two parts:-

Part (1): Self-assessment structured questionnaire developed by the researcher based on reviewing the related literature review (Mcmahon et al., 2007; Bolfíková et al., 2010; Morsy, 2010; Olmstead, 2010; Strom and Strom, 2011; Whetten and Cameron, 2011; Ahmed, 2012; Agbeh, 2015; Deep et al., 2016; Bulog et al., 2017 and Gassas et al., 2017) to determine internnurses' (experimental and control group) managerial skills practice. It divided into three categories as (conceptual, human relation and technical skills). It consisted of 65 items distributed as the follow;

a) Conceptual skills (Decision making skills): It contained 17 items to assess decision making skills of intern-nurses.

b) Human relation skills (teamwork and conflict resolution skills)

- Teamwork skills: It contained 16 items divided into three dimensions as; attends to teamwork (3 items), seeks and share information ( 6 items), and communication with teammates ( 7 items) to assess teamwork skills among intern-nurses.

- Conflict resolution skills: It consisted of 15 items that measure the five components of conflict resolution strategies such as: collaborating (3 items) founded in (1, 5, $7)$, competing (3 items) founded in (4, 9 , 12), compromising (3 items) founded in $(2,8,13)$, avoiding (3 items) founded in 
$(6,10,15)$, and accommodating (3 items) founded in $(3,11,14)$.

c) Technical skills (Time management): It contained 17 items to assess intern-nurses' managerial skills regarding time management. Scoring system:

The intern-nurses' level of managerial skills was evaluated by using a three point Likert scale as follow: (3) always (2) sometimes (1) never. The scoring system was reversed for negative items. The scores of each dimension summed up and converted into percent scores, range of scores from (65-195). Each intern-nurse had chosen one best answer after reading carefully and understanding. Finally, the answer was assigned numerical values. Accordingly scores that reflect intern-nurses' level of managerial skills as the following; skills were considered high level if the percent score was $\geq 75 \%$ that equal $\geq 146$ points, it considered moderate level if the percent score was ranged from $60-<75 \%$ that equal $117-<146$ points and it considered low level if the percent score $<60 \%$ that equal $<117$ points (Mahdy and Mahfouz, 2016).

Part (2): Managerial skills' practice by using scenarios:

It developed by the researcher based on literature review, text books and electronic text (Kelly, 2010; Whetten and Cameron, 2011; Parsons, 2015 and Dahlkemper, 2018). It consisted of four scenarios regarding managerial skills as; decision making, teamwork, conflict management, and time management to enhance managerial skills and assess problem solving ability among internnurses (experimental group). They were intended to be used in applying the steps of PBL.
Tool (IV): Attitude toward PBL questionnaire:

A Structured questionnaire developed by the researcher based on reviewing the related literature review (Alper, 2008; Rashid, 2011; Emerald et al., 2013 and Alajmi, 2014) to determine intern-nurses' (experimental group) attitude toward PBL. It included 26 items divided into five dimensions as follow; the use of PBL (8 items), learning environment with PBL (5 items), intern-nurse's peer collaboration in PBL (5 items), intern-nurse's facilitator collaboration in PBL (3 items), and the outcome of PBL (5 items) e.g. contribute to cognitive development.

Scoring system:

The studied experimental group responses were rated on a three point Likert scale as follows: always ( 3 points), sometimes ( 2 point) and never (1 point). Range of scores from (26-78). Each intern-nurse had chosen one best answer after reading carefully and understanding. Finally, the answer was assigned numerical values so, the internnurses' attitude toward PBL was categorized as the follow; Positive attitude if the percent $\geq$ $60 \%$ that equal $\geq 46$ points, negative attitude if the percent $<60 \%$ that equal $<46$ points (Ahmed, 2013 and Abd-Elmonem et al., 2019).

Tool (V): Evaluation questionnaire. It included two parts:-

Part (1): Observational checklist for intern-nurses' performance: Tutor evaluation.

It developed by the researcher based on literature review as (Valle et al., 1999; Allareddy et al., 2011 and Othman and Shalaby, 2014). It was used by tutor to evaluate certain criteria of intern-nurses' (experimental group) performance and abilities throughout the problem-based 
Enhancing Managerial Skills for Intern-Nurses by Using Problem-Based Learning Strategy

scenarios. It included 27 items divided into four dimensions as; critical appraisal (7 items), utilization of learning resources (4 items), group work (8 items), and communication skills ( 8 items).

\section{Scoring system:}

The intern-nurses' responses were measured based on a three points Likert scale as follows: always (3 points), sometimes (2 point) and never (1 point). Range of scores was (27-81). So, the level of intern-nurses performance was categorized as the following; Good performance level if the percent $\geq 75 \%$ equal to $\geq 61$ points, average performance level from $60 \%$ to less than $75 \%$ equal to $48-<61$ points and poor level $<60$ $\%$ that equal to $<48$ points (Mahdy and Mahfouz, 2016).

Part (2): Self-evaluation questionnaire. It developed by the researcher based on literature review as (Chia and Chin, 2008; Looi and Seyal, 2014 and Gönc et al., 2017.) to examine the intern-nurses' (experimental group) self-evaluation after PBL sessions. It consisted of 22 items to help intern-nurses to reflect their own performance related to knowledge-application skills (4 items) e.g. I am able to search for and access information from various sources, independent learning (7 items) e.g. identify learning objectives, communication (5 items) e.g. share my ideas clearly with the group during group discussions, and teamwork (6 items) e.g. support other members of the team.

\section{Scoring system:}

The experimental intern-nurses group responses were compared by using a threepoint Likert scale: always (3 points), sometimes (2 point) and never (1 point). Range of scores from (22-66). The level of intern-nurses performance as evaluated by themselves as follow; Good performance level if the percent $\geq 75 \%$ equal to $\geq 49$ points, average performance level from $60 \%$ to less than $75 \%$ equal to $40-<49$ points and poor level $<60 \%$ that equal to $<40$ points (Mahdy and Mahfouz, 2016).

\section{Administrative and ethical considerations}

An official permission was issued from Dean of the Faculty of Nursing Benha University to the Director of Benha University Hospital to obtain the approval for data collection to conduct the current study. Before conducting the study, the researcher explained the nature and aim of the study to intern-nurses and informed that participation in the study is voluntary. Written informal consent was obtained from each participant in the study. Confidentiality of data obtained was protected by the allocation of a code number to the questionnaire sheets. Subjects were informed that the obtained data will be used for the research purpose only. Participants' right to withdraw from the study at any time with no consequences was ascertained.

\section{The operational design}

The operational design included preparatory phase, pilot study and field work. It covered 16 months beginning from March, 2018 to the end of June, 2019.

Preparatory phase: It included the following;

Reviewing the national and international related literature using journals, periodicals, textbooks, internet and theoretical knowledge of the various aspects concerning the topic of the study to develop the current tools, scenarios and prepare the tutor for applying PBL process. This phase extended from the beginning of March, 2018 to the end of May, 2018.

Content validity: Face and content of study tools and scenarios were revised by seven 


\section{Asmaa Khaled Abd El-Aziz, Gehan Mohamed Ahmed Mostafa, Ebtesam Saeed Ahmed}

Experts' in the field of Nursing Administration; two Professors from Faculty of Nursing, Cairo and Menoufia University, five Assistant Professors from Faculty of Nursing, Ain Shams, Tanta and Menoufia University. The validity of the tools aimed to judge its clarity, comprehensiveness, relevance, simplicity, and accuracy. Based on the experts' prospective, minor modifications were done and the researcher developed the final validated form of the tools. This phase took one month June, 2018.

\section{Reliability of tools:}

Reliability of the tools was applied by using Cronbach's Alpha Coefficient test. Intern-nurses' opinionnaire sheet was $\alpha=0.946$. Managerial skills knowledge questionnaire was $\alpha=0.814$, managerial skills' practice questionnaire was $\alpha=0.921$ (regarding categories of managerial skills' practice; Conceptual skills: $\alpha=0.861$, human relation skills: $\alpha=0.907$ and technical skills: $\alpha=0.925)$, attitude toward PBL questionnaire was $\alpha=0.970$, self-evaluation questionnaire was $\alpha=0.863$ and observational checklist for intern-nurses' performance: tutor evaluation was $\alpha=0.917$. Which reflect accepted internal consistency of the tools.

\section{Pilot study:}

A pilot study was carried out from the beginning of July, 2018 to the end of July, 2018 to ascertain the clarity and applicability of the study tools and estimate the time needed for each tool. It was done on 13 intern-nurses representing $10 \%$ of total study subjects. In the light of the pilot study analysis, no modification was done and the last form was developed. So, study subjects included in the study.

\section{Field work:}

The following phases were adopted to achieve the aim of the current study: assessment, planning, implementation and evaluation phases. These phases were carried out from the earliest starting point of August, 2018 to June, 2019.

\section{Assessment phase:}

- The process of data collection was carried out in August, 2018.

- The researcher was available at the previously mentioned settings three days weekly (Saturday, Wednesday and Thursday) morning and afternoon shifts to collect baseline data.

- At the beginning, the researcher welcomed the intern-nurses, gave a brief idea about the aim and activity of the program for all intern-nurses and classified the intern-nurses into two groups (54 experimental group and 70 control group) according to their selection.

- Then, the researcher collect data for both groups by using the following questionnaire; for experimental group (Tool I, II, III, IV and V) and for control group (Tool I part 1, II and III part 1).

- The time required for finishing each questionnaire was around; 15-20 minutes for intern-nurses' opinionnaire sheet, 20-30 minutes for managerial skills knowledge questionnaire, 30-40 minutes for managerial skills' practice, 10-15 minutes for attitude toward PBL, 10-15 minutes for selfevaluation and 10-15 minutes for observational checklist of internnurses' performance. 


\section{Planning phase:}

Based on baseline data obtained from pre-test assessment and relevant review of literature, the program was developed by the researcher. This was taken two months from the beginning of September till the end of October, 2018.

- Program construction in a form of printed English form to enhance managerial skills for control group (TBL). It included different topics related to categories of managerial skills; conceptual skills (decision making), human relation skills (teamwork and conflict management) and technical skills (time management). Also, the researcher prepared power point presentation of the topics. In addition, Intern-nurses' guidelines of PBL and scenarios were prepared in a form of printed English to enhance managerial skills by using PBL strategy for experimental group (PBL group).

\section{Implementation phase:}

The implementation phase was achieved through sessions at a period of four months beginning from November, 2018 till the end of February, 2019.

\section{- For control group (TBL):}

Firstly, the researcher began the program with control group to avoid bias and interaction between TBL and PBL that may affect the results. The researchers visited each previous mentioned setting in the two shifts (morning and afternoon), three days/week. Then, the researcher divided the control group to three groups, each group composed of 20-23 intern-nurses. The educational program involved (8) theoretical sessions and were implemented according to working circumstances. These sessions were repeated with the same to each subgroup of (20-23) intern-nurses. The duration of each session lasted from 1.5-2 hours. Different methods of teaching were used such as lecture and group discussion. The teacher explained the topics, students listened and took notes. Suitable teaching media were included an educational booklet that distributed to all intern-nurses in the first day of the educational intervention.

\section{- For experimental group (PBL group):}

At the beginning, the researcher gave an orientation to the intern-nurses to provide information about PBL and problem solving skills and prepare the intern-nurses for applying them in PBL process through power point presentations, role-play and a copy of intern-nurses' guidelines of PBL was given to each group which included; definition of PBL, goal, advantages, steps, characteristics and role of intern-nurses during PBL sessions. The researcher divided the experimental group (54 intern-nurses) into 5 sub-groups and each group consisted of 10-12 intern-nurses. Each group member was given assigned different roles as; the group leader, the time keeper, and the secretary. Also, the researcher prepared the learning environment in which the study was conducted and all needed resources were prepared. The PBL tutorial sessions were held in classes equipped with; chairs (placed in a way that student could have face-to-face discussions), pens and white board/flipchart.

The problem-based learning sessions took place one day per week over 8 weeks, and each PBL scenario was conducted in 2 sessions (one session /week). The first session where the intern-nurses can identify their learning issues followed by selfdirected learning to search for the learning objectives for their identified learning issues and the second session where students discuss the acquired information among the group and solve the problem. The time for each session ranged from 1.5-2 hours. There 


\section{Asmaa Khaled Abd El-Aziz, Gehan Mohamed Ahmed Mostafa, Ebtesam Saeed Ahmed}

were four scenarios about managerial skills (decision making, teamwork, conflict management and time management) given to each group as problems, need to be solved by each group. The tutor/resarcher acted as a facilitator to guide intern-nurse learning through the learning cycle, stimulate intern-nurses towards self-directed learning, keep the learning process going and modulate the challenge of the scenario situation. While, intern-nurses follow the principles of group dynamics by appointing different roles to maintain fruitful discussion in each session. The tutor/researcher took attendance of internnurse and assessed group activity each session.

\section{Evaluation phase:}

After program implementation the post test was carried out to assess (knowledge and practice) by using the same tools of the pretest. This helped to evaluate the effect of implemented program. This was done immediately after the intervention and after 3 months (follow up) except the attitude toward PBL questionnaire was collected before and follow up phase (after 6 months). Observational checklist (tutor evaluation) was done after each session/scenario and the average was taken. The time of the data collection lasted for four months from the beginning of March, 2019 to end of June, 2019.

\section{Statistical design}

All data were collected, coded, tabulated and subjected to statistical analysis. Statistical analysis was performed by statistical Package for Social Sciences (SPSS version 20.0), also Microsoft Office Excel is used for data handling and graphical presentation. Descriptive statistics were applied in the form of mean and standard deviation for quantitative variables and frequency and percentages for qualitative variables. Qualitative categorical variables were compared using chi-square test. Pearson correlation coefficient was calculated between variables. Whenever the expected values in one or more of the cells in $2 \times 2$ tables was less than 5, Fisher exact test was used instead. Statistical significance was considered at $\mathrm{p}$-value $\mathrm{p} \leq 0.05$, and considered highly statistically significance at $\mathrm{p}$-value $\mathrm{p} \leq 0.001$. parametrical tests (e.g., independent $(\mathrm{t})$ test to compare mean scores between two sample as control and study groups, and paired(t) test to compare mean scores between the same sample at different study phases).

\section{Results:}

Table (1): Shows that all intern-nurses in both groups (PBL and TBL) had a mean of age $22.48 \pm 0.54 \& 22.63 \pm 0.62$. Regarding their gender and marital status, most of them (90.7\% \& $84.3 \%)$ were female and about two thirds $(74.1 \%$ \& $64.3 \%)$ were unmarried at PBL and TBL group respectively. As far as qualification levels before attachment to the faculty, more than two thirds of them $(77.8 \%$ \& 68.6\%) had secondary education at PBL and TBL group respectively. According to previous working experience, about three quarter of intern-nurses $(70.4 \% \& 81.4 \%)$ had no previous working experience and about one fifth $(29.6 \% \& 18.6 \%)$ had previous working experience with a mean of years $2.25 \pm 1.34 \& 1.76 \pm 1.16$ at PBL and TBL group respectively.

Figure (1): Reveals that experimental intern-nurses' opinionnaire regarding PBL or TBL at pre-program it is evident that about two thirds $(68.5 \%)$ of them preferred TBL than PBL. However, the majority of them (92.6\% \& $72.2 \%)$ preferred PBL at immediate post and follow up phases respectively than TBL as a strategy of learning. 


\section{Enhancing Managerial Skills for Intern-Nurses by Using Problem-Based Learning Strategy}

Table (2): Illustrates that more than half of studied intern-nurses $(51.9 \% \& 58.6 \%)$ at both groups PBL and TBL group at preprogram had unsatisfactory level of knowledge respectively. Meanwhile, during immediate post program phase the majority of intern-nurses $\quad(96.3 \% \quad \& \quad 87.1 \%)$ had satisfactory level of managerial skills knowledge at PBL and TBL group respectively. Also, more than three quarter (77.8\%) of PBL group and more than half $(57.2 \%)$ of TBL group had satisfactory level of managerial skills knowledge at follow up phase. It is obvious from this table that PBL group reported high satisfactory level of managerial skills knowledge than TBL group at immediate post and follow up phase. Also, there was statistical significant improvement regarding intern-nurses' managerial skills knowledge in experimental group than control group.

Figure (2): Shows that the majority of intern-nurses $(87.0 \%)$ had positive attitude toward PBL at follow up phase compared to pre-program.

Figure (3): Shows that there was highly statistical significant difference improvement in performance of intern-nurses as evaluated by tutor ( $\mathrm{P} \leq 0.001)$. The majority of internnurses $(92.6 \% \& 79.6 \%)$ had good performance at immediate post and follow up phase respectively.

Figure (4): Shows that at pre-program phase more than half $(51.9 \%)$ of intern-nurses had poor performance, however the majority of them $(90.7 \% \quad \& \quad 83.3 \%)$ had good performance at immediate post and follow up phases respectively as reported by internnurses themselves.

Table (3): Represents that at pre-program phase there was no statistical significant difference between PBL and TBL group of intern-nurses' managerial skills practice.
Meanwhile, there was highly statistical significant difference improvement $(\mathrm{P} \leq$ 0.001 ) found in the immediate post program between both groups with higher total mean scores in PBL group than TBL group during immediate post program $175.48 \pm 9.51 \&$ $161.00 \pm 10.86$ respectively. Also, there was highly statistical significant improvement $(\mathrm{P} \leq$ 0.001 ) found at follow up phase but slightly decline compared to immediate post program with high total mean score in PBL than TBL group $163.34 \pm 12.09 \quad \& \quad 139.85 \pm 15.31$ respectively. It is obvious from this table that PBL enhanced managerial skills level regarding decision making, time management, teamwork and conflict management more than TBL.

Table (4): Illustrates that there was highly statistically significant positive correlation between total managerial skills' knowledge and total managerial skills' practice at both groups $(\mathrm{P} \leq 0.001)$. This means that when managerial skills knowledge increase, managerial skills' practice will increase also.

Table (5): Reveals that, there was a highly statistically significant positive correlation between intern-nurses' managerial skills knowledge and total attitude, opinionnaire, total performance and selfevaluation scores with $\mathrm{P} \leq 0.001$ thorough program phases.

Table (6): Shows that there was highly statistically significant positive correlation between intern-nurses' managerial skills practice and total attitude, opinionnaire, total performance and self-evaluation scores with $\mathrm{P}$ $\leq 0.001$ thorough program phases. 
Table (1): Distribution of the studied intern-nurses according to their personal characteristics $(\mathbf{n}=\mathbf{1 2 4})$

\begin{tabular}{|c|c|c|c|c|}
\hline \multirow[b]{2}{*}{$\begin{array}{l}\text { Personal } \\
\text { characteristics }\end{array}$} & \multicolumn{2}{|c|}{$\begin{array}{c}\text { PBL group } \\
\text { (experimental group) } \\
(n=54)\end{array}$} & \multicolumn{2}{|c|}{$\begin{array}{c}\text { TBL group }^{\bullet \bullet} \\
(\text { control group) } \\
(n=70)\end{array}$} \\
\hline & No. & $\%$ & No. & $\%$ \\
\hline \multicolumn{5}{|l|}{ Age (years) } \\
\hline $22-$ & 29 & $\overline{53.7}$ & 31 & 44.3 \\
\hline $23-$ & 24 & 44.4 & 34 & 48.6 \\
\hline$\geq 24$ & 1 & 1.9 & 5 & 7.1 \\
\hline Mean \pm SD & \multicolumn{2}{|c|}{$22.48 \pm 0.54$} & \multicolumn{2}{|c|}{$22.63 \pm 0.62$} \\
\hline \multicolumn{5}{|l|}{ Gender } \\
\hline Male & 5 & 9.3 & 11 & 15.7 \\
\hline Female & 49 & 90.7 & 59 & 84.3 \\
\hline \multicolumn{5}{|l|}{ Marital status } \\
\hline Unmarried & 40 & 74.1 & 45 & 64.3 \\
\hline Married & 14 & 25.9 & 25 & 35.7 \\
\hline \multicolumn{5}{|c|}{ Qualification before attachment to the faculty } \\
\hline Associate degree of nursing & 12 & 22.2 & 22 & 31.4 \\
\hline Secondary school & 42 & 77.8 & 48 & 68.6 \\
\hline \multicolumn{5}{|l|}{ Previous working experience } \\
\hline Yes & 16 & 29.6 & 13 & 18.6 \\
\hline No & 38 & 70.4 & 57 & 81.4 \\
\hline \multicolumn{5}{|c|}{ If answer is yes how many years of work experience } \\
\hline $1-$ & 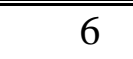 & 37.5 & $\overline{7}$ & 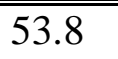 \\
\hline $2-3$ & 6 & 37.5 & 5 & 38.6 \\
\hline$\geq 4$ & 4 & 25.0 & 1 & 7.6 \\
\hline Mean \pm SD & \multicolumn{2}{|c|}{$2.25 \pm 1.34$} & \multicolumn{2}{|c|}{$1.76 \pm 1.16$} \\
\hline
\end{tabular}

- PBL: Problem Based learning

$\bullet$ TBL: Traditional Based learning 


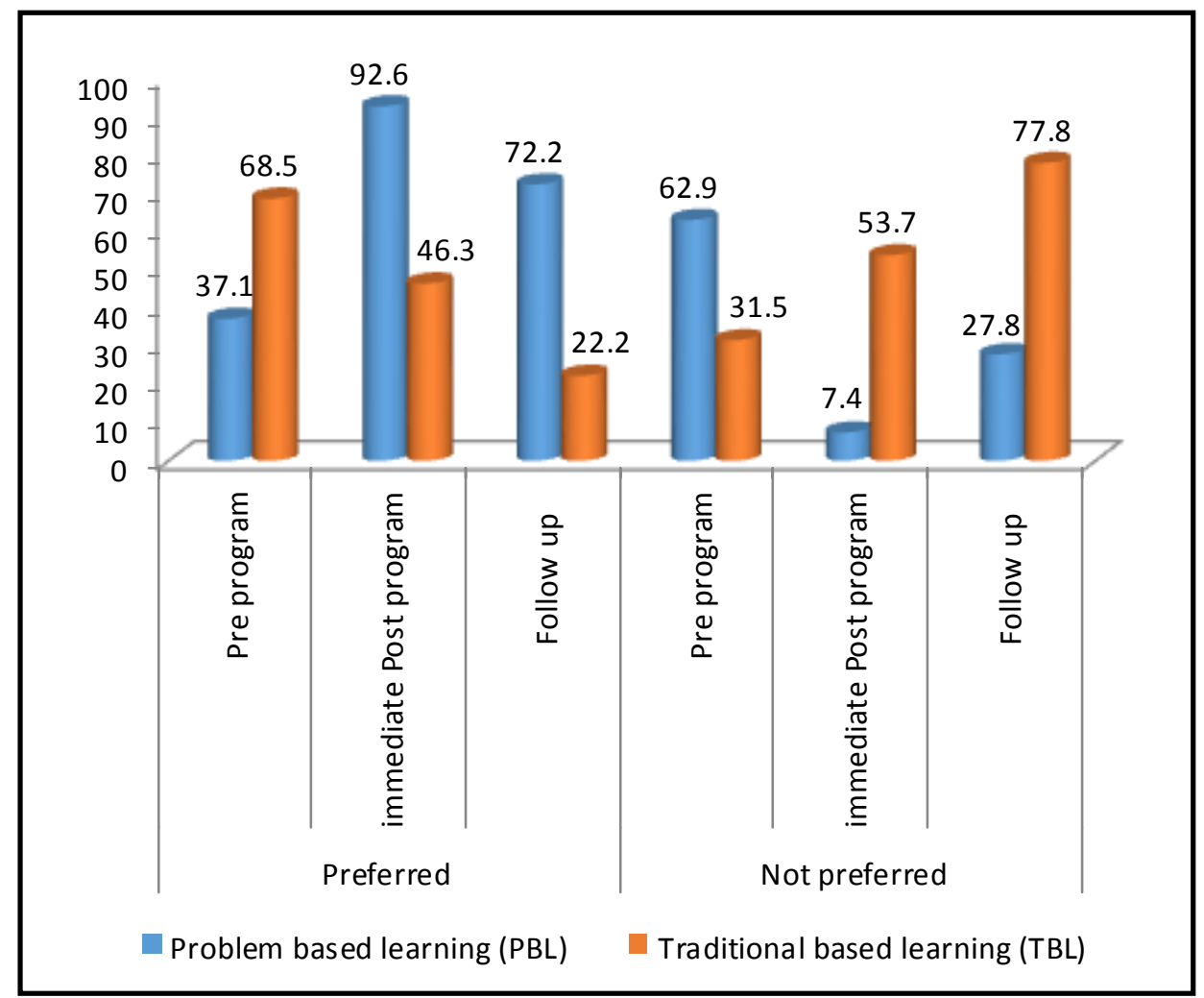

Figure (1): Total opinionnaire levels regarding PBL or TBL thorough program phases among studied experimental intern-nurses "PBL group"

Table (2): Comparison of knowledge levels regarding managerial skills between PBL and TBL group for intern-nurses thorough program phases

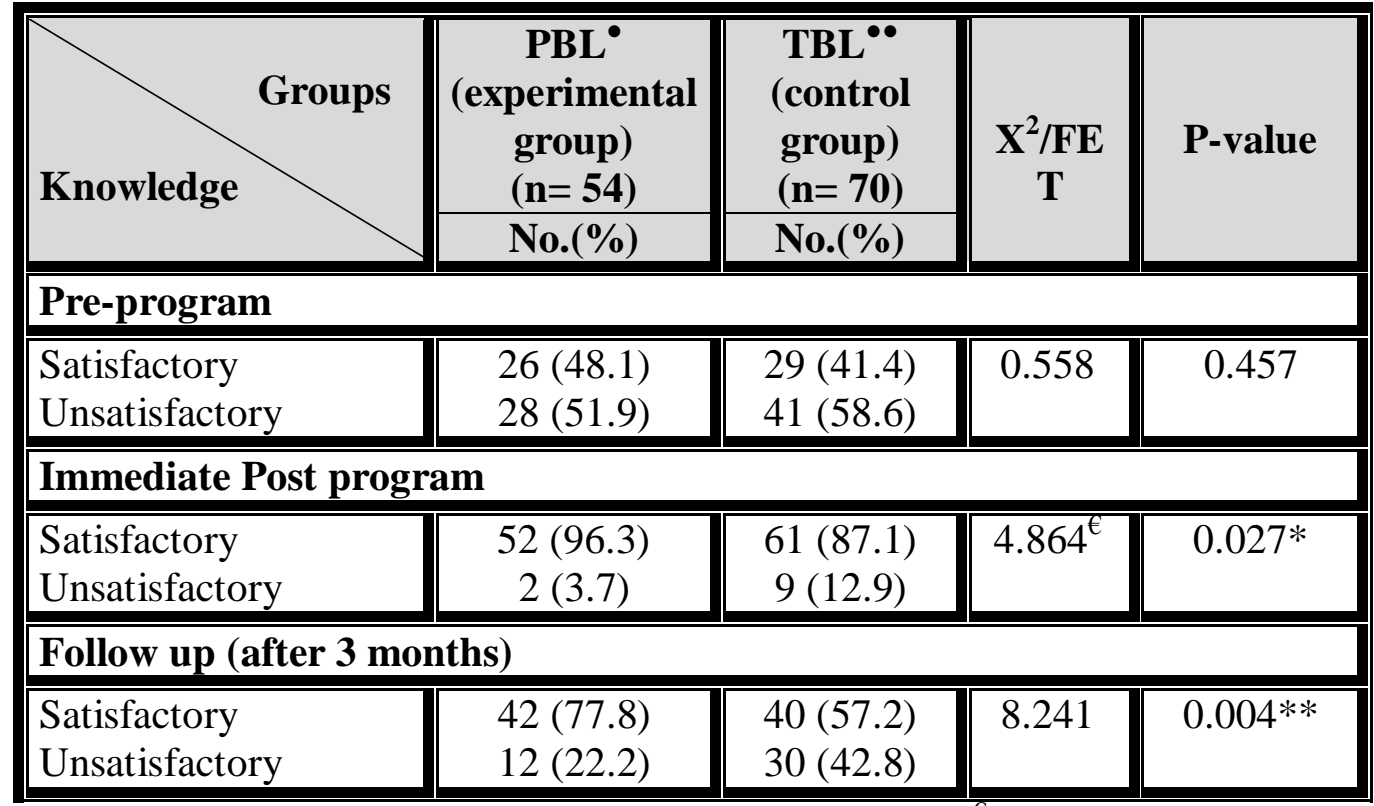

*A statistical significant difference $(\mathrm{P} \leq 0.05) \quad{ }^{\epsilon}$ Fisher Exact Test

$* *$ A highly statistical significant difference $(\mathrm{P} \leq 0.001)$

• PBL: Problem Based learning $\quad{ }^{\bullet T}$ TBL: Traditional Based learning 
Enhancing Managerial Skills for Intern-Nurses by Using Problem-Based Learning Strategy

Table (3): Mean scores and standard deviation of managerial skills' practice between PBL and TBL group among studied intern-nurses thorough program phases $(n=124)$

\begin{tabular}{|c|c|c|c|c|c|c|c|c|c|c|c|c|c|}
\hline \multirow{3}{*}{ Groups } & \multirow[t]{3}{*}{$\begin{array}{c}\text { Maximu } \\
\text { m score }\end{array}$} & \multicolumn{2}{|c|}{ Pre-program } & \multirow[t]{3}{*}{ t test } & \multirow{3}{*}{$\begin{array}{c}\text { P- } \\
\text { valu } \\
\text { e }\end{array}$} & \multicolumn{2}{|c|}{$\begin{array}{c}\text { Immediate Post } \\
\text { program }\end{array}$} & \multirow{3}{*}{$\begin{array}{c}\text { t } \\
\text { test }\end{array}$} & \multirow{3}{*}{$\begin{array}{c}\text { P- } \\
\text { value }\end{array}$} & \multicolumn{2}{|c|}{$\begin{array}{c}\text { Follow up (after } 3 \\
\text { months) }\end{array}$} & \multirow{3}{*}{$\begin{array}{c}\mathrm{t} \\
\text { test }\end{array}$} & \multirow{3}{*}{$\begin{array}{c}\text { P- } \\
\text { value }\end{array}$} \\
\hline & & $\begin{array}{c}\text { PBL }^{\bullet} \\
\text { (experime } \\
\text { ntal } \\
\text { group) } \\
(n=54)\end{array}$ & $\begin{array}{l}\text { TBL }^{\bullet \bullet} \\
\text { (control } \\
\text { group) } \\
(\mathbf{n}=70)\end{array}$ & & & $\begin{array}{c}\mathrm{PBL}^{\bullet} \\
\text { (experime } \\
\text { ntal } \\
\text { group) } \\
(\mathrm{n}=54)\end{array}$ & $\begin{array}{l}\text { TBL }^{\bullet \bullet} \\
\text { (control } \\
\text { group) } \\
(\mathbf{n}=70)\end{array}$ & & & $\begin{array}{c}\mathrm{PBL}^{\bullet} \\
\text { (experime } \\
\text { ntal } \\
\text { group) } \\
(\mathrm{n}=54)\end{array}$ & $\begin{array}{l}\text { TBL }^{\bullet \bullet} \\
\text { (control } \\
\text { group) } \\
(\mathbf{n}=70)\end{array}$ & & \\
\hline & & $\begin{array}{l}\text { Mean } \pm \\
\text { SD }\end{array}$ & Mean \pm SD & & & $\begin{array}{l}\text { Mean } \pm \\
\text { SD }\end{array}$ & Mean \pm SD & & & $\begin{array}{l}\text { Mean } \pm \\
\text { SD }\end{array}$ & Mean \pm SD & & \\
\hline $\begin{array}{l}\text { Conceptual } \\
\text { skills "Decision } \\
\text { making" }\end{array}$ & 48 & $29.19 \pm 3.18$ & $28.47 \pm 2.78$ & 1.331 & $\begin{array}{c}0.18 \\
6\end{array}$ & $\begin{array}{c}46.31 \pm 2.5 \\
8\end{array}$ & $42.06 \pm 3.10$ & $\begin{array}{c}8.14 \\
0\end{array}$ & $\begin{array}{c}0.000 * \\
*\end{array}$ & $42.83 \pm 3.79$ & $35.08 \pm 3.72$ & $\begin{array}{c}6.87 \\
0\end{array}$ & $\begin{array}{c}0.000 * \\
*\end{array}$ \\
\hline $\begin{array}{l}\text { Technical skills } \\
\text { "Time } \\
\text { management" }\end{array}$ & 51 & $25.89 \pm 6.53$ & $24.48 \pm 5.69$ & 1.276 & $\begin{array}{c}0.20 \\
4\end{array}$ & $\begin{array}{c}46.44 \pm 4.2 \\
7\end{array}$ & 43.21 \pm 5.04 & $\begin{array}{c}3.77 \\
6\end{array}$ & $\begin{array}{c}0.000 * \\
*\end{array}$ & $\overline{443.63 \pm 5.97}$ & $36.96 \pm 6.69$ & $\begin{array}{c}5.76 \\
8\end{array}$ & $\begin{array}{c}0.000 * \\
*\end{array}$ \\
\hline $\begin{array}{l}\text { Human relation } \\
\text { skills }\end{array}$ & 93 & $54.59 \pm 7.21$ & $52.20 \pm 6.87$ & 1.894 & $\begin{array}{c}0.06 \\
1\end{array}$ & $\begin{array}{c}82.72 \pm 5.1 \\
9 \\
\end{array}$ & $75.73 \pm 5.78$ & $\begin{array}{c}6.97 \\
7\end{array}$ & $\begin{array}{c}0.000 * \\
*\end{array}$ & $\overline{766.87 \pm 6.23}$ & 67.88 \pm 9.93 & $\begin{array}{c}5.82 \\
0\end{array}$ & $\begin{array}{c}0.000 * \\
*\end{array}$ \\
\hline Teamwork & 48 & $24.74 \pm 4.49$ & $23.71 \pm 4.54$ & 1.252 & $\begin{array}{c}0.21 \\
3\end{array}$ & $\begin{array}{c}44.07 \pm 3.0 \\
3 \\
\end{array}$ & $40.09 \pm 4.31$ & $\begin{array}{c}5.77 \\
6\end{array}$ & $\begin{array}{c}0.000 * \\
*\end{array}$ & $\overline{30.31 \pm 4.44}$ & $34.68 \pm 4.39$ & $\begin{array}{c}5.78 \\
6\end{array}$ & $\begin{array}{c}0.001 * \\
*\end{array}$ \\
\hline $\begin{array}{l}\text { Attends to } \\
\text { teamwork }\end{array}$ & 9 & $4.79 \pm 1.52$ & $4.54 \pm 1.39$ & 0.966 & $\begin{array}{c}0.33 \\
6\end{array}$ & $8.31 \pm 0.89$ & $7.60 \pm 1.15$ & $\begin{array}{c}3.78 \\
8\end{array}$ & $\begin{array}{l}0.000^{*} \\
*\end{array}$ & $8.39 \pm 0.83$ & $7.58 \pm 1.01$ & $\begin{array}{c}4.71 \\
7\end{array}$ & $\begin{array}{l}0.000^{*} \\
*\end{array}$ \\
\hline $\begin{array}{l}\text { Seeks and shares } \\
\text { information }\end{array}$ & 18 & $9.26 \pm 2.02$ & $8.70 \pm 1.81$ & 1.624 & $\begin{array}{c}0.10 \\
7\end{array}$ & $\begin{array}{c}16.69 \pm 1.2 \\
4\end{array}$ & $15.14 \pm 1.84$ & $\begin{array}{c}5.28 \\
9\end{array}$ & $\begin{array}{c}0.000^{*} \\
*\end{array}$ & $14.00 \pm 2.85$ & $12.64 \pm 1.85$ & $\begin{array}{c}3.20 \\
2\end{array}$ & $\begin{array}{c}0.000^{*} \\
*\end{array}$ \\
\hline Communicates & 21 & $10.68 \pm 2.19$ & $10.32 \pm 2.43$ & 0.845 & 0.40 & $19.07 \pm 2.1$ & $17.34 \pm 2.49$ & 4.03 & $0.000^{*}$ & $16.93 \pm 2.77$ & $14.60 \pm 3.21$ & 4.24 & $0.000^{*}$ \\
\hline
\end{tabular}


Asmaa Khaled Abd El-Aziz, Gehan Mohamed Ahmed Mostafa, Ebtesam Saeed Ahmed

\begin{tabular}{|c|c|c|c|c|c|c|c|c|c|c|c|c|c|}
\hline with team & & & & & 0 & 9 & & 8 & $*$ & & & 5 & $*$ \\
\hline $\begin{array}{l}\text { Conflict } \\
\text { management }\end{array}$ & 45 & $29.85 \pm 4.17$ & $28.51 \pm 4.81$ & $\begin{array}{r}1.62 \\
5\end{array}$ & $\begin{array}{c}0.10 \\
7\end{array}$ & $\begin{array}{c}38.65 \pm 3.6 \\
7\end{array}$ & $35.64 \pm 3.39$ & $\begin{array}{c}4.71 \\
9\end{array}$ & $\begin{array}{c}0.000 * \\
*\end{array}$ & $37.56 \pm 4.05$ & $33.64 \pm 5.42$ & $\begin{array}{c}4.43 \\
0\end{array}$ & $\begin{array}{c}0.000 * \\
*\end{array}$ \\
\hline Collaborating & 9 & $4.78 \pm 2.23$ & $4.61 \pm 2.07$ & $\begin{array}{r}1.65 \\
5\end{array}$ & $\begin{array}{c}0.67 \\
4\end{array}$ & $8.81 \pm .39$ & $8.23 \pm 1.12$ & $\begin{array}{c}5.77 \\
6\end{array}$ & $\begin{array}{c}0.000^{*} \\
*\end{array}$ & $7.48 \pm 1.12$ & $5.86 \pm 1.38$ & $\begin{array}{c}7.03 \\
9\end{array}$ & $\begin{array}{c}0.000 * \\
*\end{array}$ \\
\hline Competing & 9 & $6.28 \pm 0.79$ & $5.97 \pm 1.12$ & 1.714 & $\begin{array}{c}0.08 \\
9\end{array}$ & $7.48 \pm 1.13$ & $6.50 \pm 1.09$ & $\begin{array}{c}4.90 \\
4\end{array}$ & $\begin{array}{c}0.000^{*} \\
*\end{array}$ & $7.50 \pm 1.13$ & $5.98 \pm 1.91$ & $\begin{array}{c}5.17 \\
5\end{array}$ & $\begin{array}{c}0.000 * \\
*\end{array}$ \\
\hline Avoiding & 9 & $8.29 \pm 1.01$ & $8.08 \pm 1.05$ & 1.132 & $\begin{array}{c}0.26 \\
0\end{array}$ & $7.22 \pm 1.78$ & $6.13 \pm 1.85$ & $\begin{array}{l}3.32 \\
2\end{array}$ & $\begin{array}{c}0.001^{*} \\
*\end{array}$ & $6.98 \pm 1.54$ & $5.70 \pm 2.02$ & $\begin{array}{c}3.87 \\
0\end{array}$ & $\begin{array}{c}0.000 * \\
*\end{array}$ \\
\hline Accommodating & 9 & $5.31 \pm 1.58$ & $5.16 \pm 1.65$ & 0.537 & $\begin{array}{c}0.59 \\
2\end{array}$ & $8.00 \pm 1.18$ & $7.27 \pm 1.02$ & $\begin{array}{c}3.67 \\
9\end{array}$ & $\begin{array}{c}0.000^{*} \\
*\end{array}$ & $8.09 \pm 1.10$ & $6.26 \pm 1.85$ & $\begin{array}{c}6.46 \\
3\end{array}$ & $\begin{array}{c}0.000 * \\
*\end{array}$ \\
\hline Compromising & 9 & $5.41 \pm 1.84$ & $5.01 \pm 1.94$ & 1.145 & $\begin{array}{c}0.25 \\
4\end{array}$ & $8.19 \pm 0.89$ & $7.59 \pm 0.89$ & $\begin{array}{c}3.70 \\
9\end{array}$ & $\begin{array}{c}0.000^{*} \\
*\end{array}$ & $7.56 \pm 1.34$ & $5.97 \pm 1.73$ & $\begin{array}{c}5.56 \\
6\end{array}$ & $\begin{array}{c}0.000 * \\
*\end{array}$ \\
\hline Total & 192 & $\begin{array}{c}109.67 \pm 14 \\
47\end{array}$ & $\begin{array}{c}105.60 \pm 12 . \\
76\end{array}$ & 1.660 & $\begin{array}{c}0.10 \\
0\end{array}$ & $\begin{array}{c}175.48 \pm 9 . \\
51 \\
\end{array}$ & $\begin{array}{c}161.00 \pm 10.8 \\
6\end{array}$ & $\begin{array}{c}7.76 \\
7 \\
\end{array}$ & $\begin{array}{c}0.000 * \\
*\end{array}$ & $\begin{array}{c}163.34 \pm 12 . \\
09\end{array}$ & $\begin{array}{c}139.92 \pm 15.3 \\
1\end{array}$ & $\begin{array}{c}8.27 \\
6 \\
\end{array}$ & $\begin{array}{c}\mathbf{0 . 0 0 0} * \\
*\end{array}$ \\
\hline
\end{tabular}

**A highly statistical significant difference $(\mathrm{P} \leq 0.001) \quad \mathrm{t}$ test: Independent $\mathrm{t}$ test

- PBL: Problem Based learning

$\bullet$ TBL: Traditional Based learning 


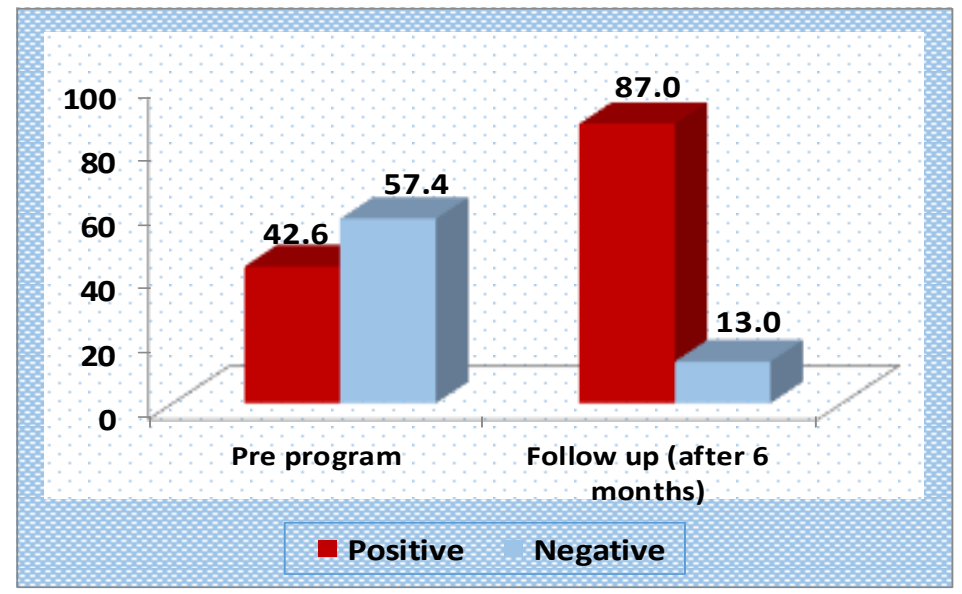

Figure (2): Total levels of attitude toward PBL among studied PBL group at pre-program and follow up

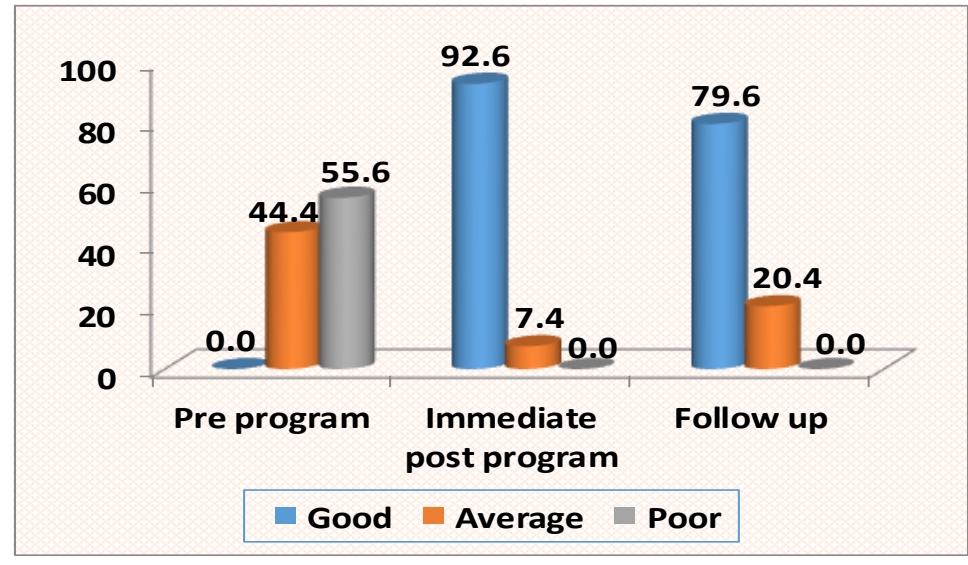

Figure (3): Total levels of intern-nurses' performance thorough program phases: tutor evaluation

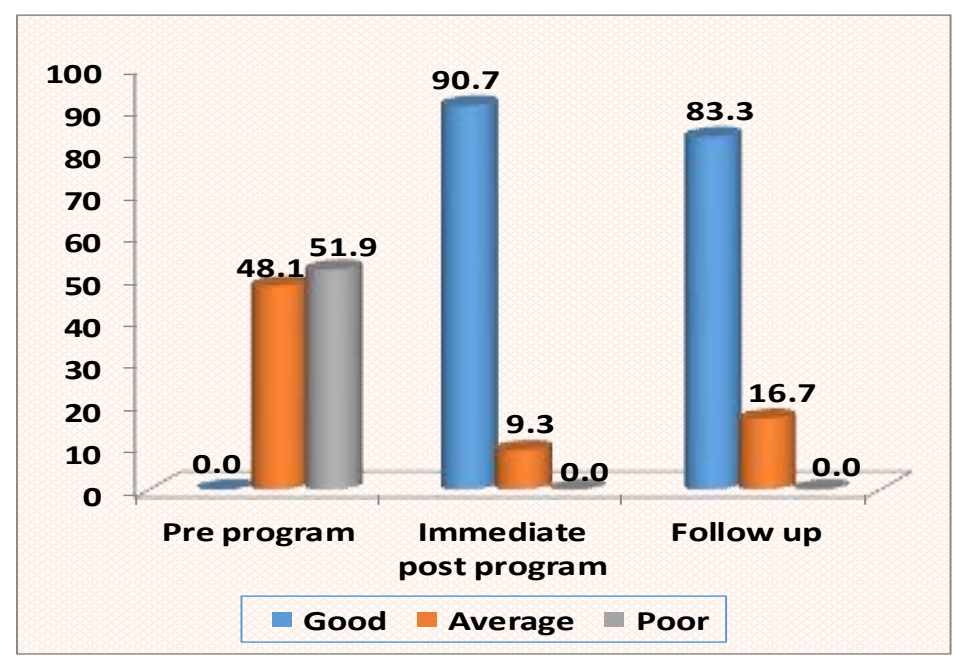

Figure (4): Total self-evaluation levels among studied PBL group thorough program phases 
Table (4): Correlations coefficient between total knowledge and managerial skills scores among PBL and TBL group thorough program phases $(n=124)$

\begin{tabular}{|c|c|c|c|c|c|c|c|c|c|c|c|c|}
\hline \multirow{4}{*}{ Groups } & \multicolumn{12}{|c|}{ Total managerial skills' knowledge scores $(n=124)$} \\
\hline & \multicolumn{4}{|c|}{ Pre-program } & \multicolumn{4}{|c|}{ immediate Post program } & \multicolumn{4}{|c|}{ Follow up (after 3 months) } \\
\hline & \multicolumn{2}{|c|}{$\begin{array}{c}\mathrm{PBL}^{\bullet} \\
\text { (experiment } \\
\text { al group) } \\
(\mathrm{n}=54)\end{array}$} & \multicolumn{2}{|c|}{$\begin{array}{l}\text { TBL }^{\bullet \bullet} \\
\text { (control } \\
\text { group) } \\
(\mathrm{n}=70)\end{array}$} & \multicolumn{2}{|c|}{$\begin{array}{c}\mathrm{PBL}^{\bullet} \\
( \\
\text { experimenta } \\
\text { I group) } \\
(\mathrm{n}=54) \\
\end{array}$} & \multicolumn{2}{|c|}{$\begin{array}{l}\mathrm{TBL}^{\bullet \bullet} \\
\text { (control } \\
\text { group) } \\
(\mathrm{n}=70)\end{array}$} & \multicolumn{2}{|c|}{$\begin{array}{c}\mathrm{PBL}^{\circ} \\
( \\
\text { experimenta } \\
1 \text { group) } \\
(\mathrm{n}=\mathbf{5 4})\end{array}$} & \multicolumn{2}{|c|}{$\begin{array}{l}\text { TBL }^{\bullet \bullet} \\
\text { (control } \\
\text { group) } \\
(n=70)\end{array}$} \\
\hline & $\overline{\mathbf{r}}$ & $\begin{array}{c}\mathbf{P} \\
\text { value }\end{array}$ & $\overline{\mathbf{r}}$ & $\begin{array}{c}P \\
\text { value }\end{array}$ & $\overline{\mathbf{r}}$ & $\begin{array}{c}\mathbf{P} \\
\text { value }\end{array}$ & $\overline{\mathbf{r}}$ & P value & $\overline{\mathbf{r}}$ & $\begin{array}{c}\mathbf{P} \\
\text { value }\end{array}$ & $\overline{\mathbf{r}}$ & $\begin{array}{c}\mathbf{P} \\
\text { value }\end{array}$ \\
\hline $\begin{array}{l}\text { Total managerial } \\
\text { skills' practice } \\
\text { scores }\end{array}$ & $\begin{array}{c}0.42 \\
7\end{array}$ & $\begin{array}{c}\mathbf{0 . 0 0 2} \\
* *\end{array}$ & $\begin{array}{c}0.36 \\
6\end{array}$ & $\begin{array}{c}\mathbf{0 . 0 0 3} \\
* *\end{array}$ & $\begin{array}{c}0.84 \\
7\end{array}$ & $\begin{array}{c}0.000 \\
* *\end{array}$ & $\begin{array}{c}0.73 \\
4\end{array}$ & $\begin{array}{c}0.000 * \\
*\end{array}$ & $\begin{array}{c}0.58 \\
6\end{array}$ & $\begin{array}{c}0.000 \\
* *\end{array}$ & $\begin{array}{c}0.53 \\
2\end{array}$ & $\begin{array}{c}\mathbf{0 . 0 0 0} \\
* *\end{array}$ \\
\hline
\end{tabular}

**A highly statistical significant difference $(\mathrm{P} \leq 0.00)$

- PBL: Problem Based learning

• TBL: Traditional Based learning 
Table (5): Correlations coefficient between total knowledge and studied variables scores among PBL group thorough program phases $(n=54)$

\begin{tabular}{|c|c|c|c|c|c|c|}
\hline \multirow{3}{*}{ Variables } & \multicolumn{6}{|c|}{ Total managerial skills' knowledge scores } \\
\hline & \multicolumn{2}{|c|}{$\begin{array}{l}\text { Pre-program } \\
\quad(n=54)\end{array}$} & \multicolumn{2}{|c|}{$\begin{array}{l}\text { immediate Post } \\
\text { program } \\
(n=54)\end{array}$} & \multicolumn{2}{|c|}{$\begin{array}{c}\text { Follow up } \\
(n=54)\end{array}$} \\
\hline & $\mathbf{r}$ & $P$ value & $\mathbf{r}$ & P value & $\mathbf{r}$ & P value \\
\hline Total attitude score & 0.416 & $0.000 * *$ & - & - & 0.453 & $0.000^{* *}$ \\
\hline Total opinionnaire score & 0.323 & $0.000 * *$ & 0.369 & $0.000 * *$ & 0.364 & $0.000 * *$ \\
\hline $\begin{array}{l}\text { Total student evaluation } \\
\text { score (tutor evaluation) }\end{array}$ & 0.637 & $0.000 * *$ & 0.612 & $0.000 * *$ & 0.557 & $0.000^{* *}$ \\
\hline $\begin{array}{l}\text { Total Self-evaluation } \\
\text { score }\end{array}$ & 0.663 & $0.000 * *$ & 0.514 & $0.000 * *$ & 0.570 & $0.000 * *$ \\
\hline
\end{tabular}

*A statistical significant difference $(\mathrm{P} \leq 0.05) \quad * *$ A highly statistical significant difference $(\mathrm{P} \leq$ $0.001)$

Table (6): Correlations coefficient between total managerial skills and studied variables scores

\begin{tabular}{|c|c|c|c|c|c|c|}
\hline \multirow{3}{*}{ Variables } & \multicolumn{6}{|c|}{$\overline{~ T o t a l}$ managerial skills' practice scores } \\
\hline & \multicolumn{2}{|c|}{$\begin{array}{l}\text { Pre-program } \\
\quad(n=54)\end{array}$} & \multicolumn{2}{|c|}{$\begin{array}{c}\text { immediate Post } \\
\text { program } \\
(n=54)\end{array}$} & \multicolumn{2}{|c|}{$\begin{array}{l}\text { Follow up } \\
(n=54)\end{array}$} \\
\hline & $\mathbf{r}$ & $P$ value & $\mathbf{r}$ & P value & $\mathbf{r}$ & $P$ value \\
\hline Total attitude score & 0.287 & $0.021^{*}$ & - & - & 0.327 & $0.000 * *$ \\
\hline $\begin{array}{l}\text { Total opinionnaire } \\
\text { score }\end{array}$ & 0.652 & $0.000 * *$ & 0.563 & $0.000 * *$ & 0.620 & $0.000 * *$ \\
\hline $\begin{array}{l}\text { Total student } \\
\text { evaluation score } \\
\text { (tutor evaluation) }\end{array}$ & 0.627 & $0.000 * *$ & 0.649 & $0.000 * *$ & 0.672 & $0.000 * *$ \\
\hline $\begin{array}{l}\text { Total Self- } \\
\text { evaluation score }\end{array}$ & 0.684 & $0.000 * *$ & 0.613 & $0.000 * *$ & 0.771 & $0.000 * *$ \\
\hline
\end{tabular}

among PBL group thorough program phases $(n=54)$

*A statistical significant difference $(\mathrm{P} \leq 0.05) \quad * *$ A highly statistical significant difference $(\mathrm{P} \leq$ $0.001)$ 


\section{Discussion}

Quality in nursing practice depends upon educational preparation of nurses to solve problem, think critically, and make decisions in today's health care system. Therefore, using problem based learning (PBL) is very important to empower students' work through actively participating in the learning process, and identify learning goals with peers, engaging in self-study. Also, PBL empower students' skills through discussing and applying new learning strategy, and integrating a variety of knowledge (Soliman et al., 2017).

The present study aimed to enhance managerial skills for intern-nurses by using problem based learning strategy at Benha University Hospital through; determining intern-nurses' opinion regarding traditional methods or problem-based learning, assessing intern-nurses' knowledge and skills regarding managerial skills, identifying intern-nurses' attitude toward problem-based learning, designing and implementing managerial skills program for intern-nurses by using problembased learning strategy and evaluating the effectiveness of the designed program on intern-nurses' managerial knowledge and skills.

Discussion of the present study is presented in the following sequences; Part I: Personal characteristics of the studied internnurses; Part II: Intern-nurses' opinionnaire regarding problem based learning (PBL) or traditional based learning (TBL) thorough program phases; Part III: Intern-nurses' managerial skills knowledge thorough program phases; Part IV: Managerial skills' practice among studied intern-nurses between PBL and TBL group; Part V: Attitude toward PBL among studied PBL group at pre and follow up phases; Part VI: Evaluation of studied PBL group thorough program phases and Part VII: Correlations among studied variables at different study phases.

\section{Part I. Personal characteristics of the studied intern-nurses}

Concerning personal characteristics of intern- nurses, the results of the present study showed that all intern-nurses in both groups (PBL and TBL) had a mean of age $22.48 \pm$ $0.54 \& 22.63 \pm 0.62$. Regarding their gender and marital status, most of them were female and about two thirds were unmarried at PBL and TBL group respectively. As far as qualification levels before attachment to the faculty, more than two thirds of them had secondary education at PBL and TBL group respectively. According to previous working experience, about three quarter of internnurses had no previous working experience and about one fifth had previous working experience with a mean of years $2.25 \pm 1.34$ $\& 1.76 \pm 1.16$ at $\mathrm{PBL}$ and TBL group respectively.

\section{Part II. Intern-nurses' opinionnaire regarding problem based learning (PBL) or traditional based learning (TBL) thorough program phases}

This part achieves the first study objective which was determining internnurses' opinion regarding traditional methods or problem-based learning.

Concerning Intern-nurses' opinionnaire regarding PBL or TBL, the results of the present study revealed that about two thirds of experimental intern-nurses at pre-program preferred TBL than PBL. However, the majority of them preferred PBL at immediate post and follow up phases than TBL as a strategy of learning. This might be due to PBL better for linking the knowledge of basic and 
Asmaa Khaled Abd El-Aziz, Gehan Mohamed Ahmed Mostafa, Ebtesam Saeed Ahmed

clinical sciences, a higher quality of education, knowledge retention, class attractiveness, and practical use. Also, for the advantages of PBL strategy as; increasing the ability to solve real problems, motivation boost in learning, discover own talent and the ability to work effectively in a team.

The result of present study was supported with Kim, (2019) who conducted a study entitled "Observational application comparing problem-based learning with the conventional teaching method for clinical acupuncture education" and demonstrated that PBL is preferable for clinical acupuncture education over the conventional method. Also, Dhami et al., (2017) who conducted a study about "Student's attitude toward Lbl versus $\mathrm{Pbl}$ in Pharmacology at Ngmc, Banke, Nepal" and found that student perception towards PBL was significantly higher than lecture based learning (LBL), which was found to be statistically significant showing student like PBL than LBL.

Moreover, Imran et al., (2015) who conducted a study about "Problem-based learning versus lecture-based learning in pharmacology in a junior doctor teaching program: a crossover study from northern India", Ibrahim et al., (2014) who conducted a study about "Medical students' preference of problem-based learning or traditional lectures in King Abdulaziz University" and Khoshnevisasl et al., (2014) who conducted a study entitled "Comparison of problembased learning with lecture-based learning" and found that students preferred PBL over traditional lectures/LBL.

Furthermore, Sharma, (2015) who conducted a study about "Effect of problem based learning on nursing students' clinical decision making and learning satisfaction" and showed that the students were more satisfied with the PBL method of teaching as compare to conventional didactic lecture. Besides, Banu et al., (2014) who conducted a study entitled "Problem based learning in Medical education- a review" and showed a clear preference for PBL over the conventional curriculum for the interactive resource sessions and group discussions. Both students and teachers found PBL rewarding.

In the same line, Mohan et al., (2014) who conducted a study entitled "Students' attitude toward didactic lecture versus problem-based learning in pharmacology: a questionnaire based study" and found the score for PBL was significantly higher than LBL showing that students liked PBL more than LBL. Likewise, Qalawa and Keshk, (2014) who conducted a study about "Impact of problem based learning on acquiring 21 century skills among nursing students (comparative study)" and discovered that Egyptian nursing students were satisfied of course teaching by PBL.

On the other hand, the result of the present study disagreement with Zeng and Wang, (2018) who conducted a study entitled "The application research of PBL and LBL in English teaching in Medical Colleges" and found that most students prefer the combination of PBL and LBL, which can greatly improve students' English proficiency, stimulate students' interest in learning English, and improve students' oral English, listening ability, and the ability to collect and collate the learning materials. Also, Padmanabha et al., (2016) who conducted study entitled "Student's perception towards learning medical sciences: problem based learning versus lecture based learning methods" and illustrated that students preferred PBL over LBL. However, the difference was not statistically significant. Although PBL edged over LBL, but most of 
Enhancing Managerial Skills for Intern-Nurses by Using Problem-Based Learning Strategy

them preferred integrated teaching going side by side, PBL along with LBL.

\section{Part III. Intern-nurses' managerial skills knowledge thorough program phases}

This part answers the second study objective which was assessing intern-nurses' knowledge and skills regarding managerial skills.

Concerning intern-nurses' managerial skills knowledge, the result of the present study illustrated that more than half of studied intern-nurses at both groups PBL and TBL group at pre-program had unsatisfactory level of knowledge. Meanwhile, during immediate post program phase the majority of internnurses had satisfactory level of managerial skills knowledge at PBL and TBL group. Also, more than three quarter of PBL group and more than half of TBL group had satisfactory level of managerial skills knowledge at follow up phase. It was obvious that PBL group reported high satisfactory level of managerial skills knowledge than TBL group at immediate post and follow up phase. Also, there was statistical significant improvement regarding intern-nurses' managerial skills knowledge in experimental group than control group.

From the researcher point of view, this might be due to that PBL allows participation in the learning process, communication skills, self-directed learning, reasoning skills, identifying strengths and weaknesses, learning in context, critical thinking skills and exploration of different resource materials, groups skills, made topic more interesting and fun learning imparted better content knowledge and was more effective in fulfilling learning objectives. This ultimately leads to increased motivation to learn among nursing students and enhance knowledge acquisition and retention.

This result aligns with, Kim, (2019) who demonstrated that PBL is an effective approach, especially for the retention and application of clinical acupuncture knowledge. Also, Orji and Ogbuanya, (2018) who conducted a study entitled "Assessing the effectiveness of problembased and lecture-based learning environments on students' achievements in electronic works" and revealed that PBL significantly improved the achievement of students' electronic works in the treatment group at post treatment and follow-up assessments, whereas no effect was observed in the control group at the same time points.

Furthermore, Kartikeyan and Malgaonkar, (2018) who conducted a study entitled "Comparison of cognitive domain scores after lecture-based and problem-based learning in community medicine" and found that the difference in cognitive domain scores after LBL (determined by pre-test) and PBL (determined by post-test) was highly significant. That is problem-based learning offers a standardized method of teaching wherein the knowledge can be applied.

Besides, Yadav et al., (2018) who conducted a study entitled "Attitude and perception of undergraduate medical students toward the problem-based learning in Chitwan Medical College, Nepal" and showed that PBL sessions were considered effective in improving students' professional knowledge. Moreover, AbdRabou, (2017) who conducted a study entitled "Problem based learning for improving group dynamic: Nursing students self report and perception" and illustrated that BL was beneficial learning method as it was effective in construction of student's practiced knowledge. 


\section{Asmaa Khaled Abd El-Aziz, Gehan Mohamed Ahmed Mostafa, Ebtesam Saeed Ahmed}

Further, Ahamad et al., (2017) who conducted a study entitled "Implementation of problem-based learning in geometry lessons" and revealed positive influences of PBL on students' performance in learning geometry as gain and retention of knowledge was observed. Also, Dhami et al., (2017) found that PBL stimulates students' interest, improve retention of knowledge which helps in solving questions rationally and help to apply knowledge to understand and integrating basics with clinical sciences.

Moreover, Gönc et al., (2017) who conducted a study entitled "Experience of problem-based learning for raising quality of nursing study" and found that problem-based learning stimulates the students' sense for a responsible acquiring of professional knowledge and helps to acquire knowledge to function in nursing. Likewise, Molande et al., (2017) who conducted a study entitled "Effectiveness of problem-based learning for technical teacher training in woodwork at a Malawi college" and indicated that students who learned through PBL outperformed the group that learned through traditional approach in content acquisition, knowledge interpretation, application of concepts and principles and analytical skills.

Also, Sathya and Reddemma, (2017) who conducted a study entitled "Development of problem based learning training module in nursing education" and indicated that PBL workshop was found to be effective in terms of improvement in the mean post-test knowledge score compared to the mean pretest knowledge score though it was not statistically significant.

Additionally, Sayyah et al., (2017) who conducted a study about "Use of a problem-based learning teaching model for undergraduate medical and nursing education: a systematic review and meta-analysis" and showed that using PBL had a positive effect on the academic achievement of undergraduate medical courses as academic achievement was relatively higher in PBL method compared to traditional methods such as LBL. Also, Sharma and Sachdeva, (2017) who conducted a study about "Students' perception to introduction of problem-based learning modules in Anatomy" and found that PBL assist to gain the desired knowledge incumbent of medical graduate.

As well, Abd Rahman et al., (2016) who conducted a study entitled "The impacts of problem-based learning approach in enhancing critical thinking skills to teaching literature" and showed that PBL approach has many positive impacts that are useful for learning the content knowledge in a brand new way, and it can be adapted to suit students' learning styles.

In the same context, Assumpção et al., (2016) who conducted a study entitled "Using problem-based learning in entrepreneurship education" and showed that the students started to acquire the knowledge that was required for managing business. Similarly, Augusthy, (2016) who conducted a study entitled "A comparative study of the learning outcomes and students' satisfaction from problem-based learning and lecturebased learning in obstetrics and gynaecology" and found that in comparison to LBL, the PBL method produces better outcomes of recall, analytical and critical thinking in addition to problem solving skills.

In the same line, Jindal et al., (2016) who conducted a study entitled "Pros and cons of problem-based learning in medical education: Students' viewpoint" and found that PBL strategy helped in enhancement of knowledge. In addition, Wang et al., (2016) 
Enhancing Managerial Skills for Intern-Nurses by Using Problem-Based Learning Strategy

who conducted a study entitled "Assessing the effectiveness of problem-based learning in physical diagnostics education in China: a meta-analysis" and showed that the students in the PBL groups have better knowledge scores than those in the traditional teaching method groups.

As well, Yi, (2016) conducted a study entitled " International Nursing Students' experiences of problem-based learning in a university of applied sciences" and found that compared with traditional teaching method, PBL could be utilized as learning method for nursing students to explore more knowledge independently and simultaneously promote other learning skills. Moreover, Yew and Goh, (2016) who conducted a study entitled "Problem-based learning: An overview of its process and impact on learning" and concluded that the studies comparing the relative effectiveness of PBL are generally consistent in demonstrating its superior efficacy for longer-term knowledge retention and in the application of knowledge.

Also, Agbeh, (2015) who conducted a study about "Problem-based learning in a hospitality and tourism administration course" and showed that there was a significant difference between the content knowledge mean of the pre- and post-content knowledge test after teaching the students using PBL. As well, Hande et al., (2015) who conducted a study about "Acquisition of knowledge, generic skills and attitudes through problembased learning: Student perspectives in a hybrid curriculum" and Hung and Lin, (2015) who conducted a study entitled "Using concept mapping to evaluate knowledge structure in problem-based learning" and indicated that PBL had a strong effect on the acquisition and integration of knowledge
Likewise, Ding et al., (2014) who conducted a study entitled "Assessing the effectiveness of problem-based learning of preventive medicine education in China", Ghimire and Bhandary, (2014) who conducted a study entitled "Students' perception and preference of problem lased learning during introductory course of a Nepalese Medical School" and Mohan et al., (2014) found that PBL improved retention of knowledge and helped students to apply their knowledge and understand its relevance to a clinical scenario more than LBL.

Also, EL-Shaer and Gaber, (2014) who conducted a study about "Impact of problem-based learning on students' critical thinking dispositions, knowledge acquisition and retention" and found increasing of total mean score of knowledge acquisition (immediately post intervention) and retention (3 months post intervention) of the experimental group than total mean score of knowledge acquisition and retention of control group. Also increasing mean score of knowledge acquisition than mean score of knowledge retention in both group.

Furthermore, Frambach, (2012) who conducted a study entitled "Rethinking the globalization of problem-based learning: how culture challenges self-directed learning" and reported that PBL is the best for nursing students which tend to better acquire scientific conceptions, integrate and organize the knowledge. PBL promote both the acquisition of content knowledge and the development of thinking skills and strategies. Also, Dissanayaka et al., (2012) who conducted a study about "Physiotherapy students' perception on problem based learning" and demonstrated that PBL is an effective way of improving critical thinking, enhancing teamwork and acquiring better 
Asmaa Khaled Abd El-Aziz, Gehan Mohamed Ahmed Mostafa, Ebtesam Saeed Ahmed

factual knowledge of musculoskeletal physiotherapy.

On the other hand, this result of the present study disagreement with Tayyeb, (2013) who conducted study about "Effectiveness of problem based learning as an instructional tool for acquisition of content knowledge and promotion of critical thinking among medical students" and found that teaching through traditional method significantly improved content knowledge, but did not considerably improve clinical reasoning and problem solving skills whereas, content knowledge of students who studied through PBL remained the same.

In addition, Pourshanazari et al., (2013) who conducted a study entitled "Comparing the long-term retention of a physiology course for medical students with the traditional and problem-based learning" and showed that students who have experienced PBL achieve similar or less learning gains when it comes to short-term knowledge acquisition when compared to students in lecture-based learning environment. Moreover, Yaqinuddin, (2013) who conducted a study entitled "Problembased learning as an instructional method" and concluded that PBL does not impact knowledge acquisition, but impacts application of knowledge.

Also, Almasoudi, (2012) who conducted a study about "Problem-based learning as a teaching method versus lecturebased teaching in respiratory therapy education" and showed PBL to be effective, but not significantly more effective than traditional lecture-based methods in regards to objective test scores. As well, De Witte and Rogge, (2012) who conducted a study entitled "Problem-based learning in secondary education: Evaluation by a randomized experiment" and indicated that students in PBL course do not obtain higher test results than students in a traditional taught course. On the contrary, the estimated effect turned out to be non-significant negative effect on student achievements.

\section{Part IV. Managerial skills' practice among studied intern-nurses between PBL and TBL group}

This part answers the second study objective which was assessing intern-nurses' knowledge and skills regarding managerial skills.

Concerning intern-nurses' managerial skills practice, the results of the current study revealed that at pre-program phase there was no statistical significant difference between PBL and TBL group of intern-nurses' managerial skills practice. Meanwhile, there was highly statistical significant difference improvement found in the immediate post program between both groups with higher total mean scores in PBL group than TBL group during immediate post program. Also, there was highly statistical significant improvement found at follow up phase but slightly decline compared to immediate post program with high total mean score in PBL than TBL group. It is evident that PBL enhanced managerial skills level regarding decision making, teamwork, conflict management and time management more than TBL.

From the researcher point of view, this might be due to that intern-nurses in PBL group assigned different rotatory roles as team leader, secretary and time keeper, spent more time on group work, reference analysis capitalize on one another's resources and skills, asking one another for information, evaluating one another's ideas, and monitoring one another's work than those 
Enhancing Managerial Skills for Intern-Nurses by Using Problem-Based Learning Strategy attending conventional lectures. The nursing students find their own roles and responsibilities in the group work and independent work which helped them to acquire and enhance their managerial skills' practice.

This result was in congruence with Heaviside et al., (2018) who conducted a study entitled "Bridging the gap between education and employment: A case study of problem-based learning implementation in postgraduate sport and exercise psychology" and found that PBL was instrumental for developing key employability skills: team working, communication, thinking critically, creatively and flexibly; for helping students translate academic knowledge into application in future employment contexts, and for increasing awareness that learning is a lifelong developmental process.

Also, Yadav et al., (2018) and AbdRabou, (2017) found that BL improved students' problem-solving skills, added to their self-directed learning skills along with enriched their teamwork experience and improved their' group collaboration skills and related competences. As well, Gönc et al., (2017) found that PBL encourages nursing students' motivation, independence, communication skills and teamwork and helps to acquire skills necessary to function in nursing.

Moreover, Sharma and Sachdeva, (2017) found that PBL assist to inculcate skills, such as communication, critical thinking, logical and analytical approach to problems, reasoned decision making, and selfevaluation. Likewise, Assumpção et al., (2016) showed that after using PBL, the students started to acquire the skills and attitudes that were required for managing business by practically solving a problem.
Moreover, there has been a transformation in their ways of thinking, acting and observing the dynamics of the entrepreneurship and innovation universe. Also, the students are encouraged to show leadership sprit and management skills in acting in teams, abilities which are basic requirements to any entrepreneur.

Also, the finding was matching with Faisal et al., (2016) who conducted a study entitled "Problem-based learning in comparison with lecture-based learning among medical students" and Alarcon and Prezotto, (2016) who conducted a study entitled "Evaluation of educational strategy, grounded on problem-based learning on nursing undergraduate" and found that students in the experimental PBL group showed better performance when compared to students from the traditional method.

Likewise, Yi, (2016) found that compared with traditional teaching method, PBL has its dominant position to assist the students to prepare for the learning skills and capacity acquired in the future clinical working situations. Moreover, Alrahlah, (2016) who conducted a study entitled "How effective the problem-based learning (PBL) in dental education. A critical review" and Tian et al., (2013) and showed that students in PBL courses exhibit superior professional skills and effective learning compared with those instructed using traditional approaches. As well, Hande et al., (2015) found that PBL affected not only typical competence in interpersonal and cognitive domains but also more general work-related skills considered important for success in professional practice.

Furthermore, Wright et al., (2014) who conducted a study entitled "Problembased learning: an exploration of student opinions on its educational role in one UK 
Asmaa Khaled Abd El-Aziz, Gehan Mohamed Ahmed Mostafa, Ebtesam Saeed Ahmed

pharmacy undergraduate curriculum" and found that the majority of students agreed that PBL improved their team working, oral communication and problem-solving skills. Additionally PBL improved students' ability to identify and address ethical dilemmas as well as enhancing their ability to manage their own learning.

Besides, Yew and Goh, (2016) and Strobel and van Barneveld, (2009) found that PBL is significantly more effective than traditional approaches to train competent and skilled practitioners and to promote skills acquired during the learning experience or training session. Also, Ibrahim et al., (2014) and Meo, (2013) found that the mean total skill gained from PBL was much higher than that of lectures, and a highly statistical significant difference was observed. As well, Agbeh, (2015) indicated that PBL helps students to build a capacity for self-directed learning, foster teamwork, improve their communications skills, manage their learning time table, be active learners, find relevant and valuable information, and apply problemsolving skills.

Furthermore, Bell, (2012) who conducted a study entitled "Introducing problem-based learning as a learning strategy for Masters Students" and found that PBL seemed to have worked well with development in personal and professional skills, team working skills. Many students also acknowledged the benefits of this style of learning and the additional skills they have developed such as presentation skills, critical thinking abilities and problem solving skills.

In the same line, Ansari et al., (2015) who conducted a study entitled "Problem based learning (PBL): A novel and effective tool of teaching and learning" and found that PBL process have proved to enhance the soft skill, cognitive, metacognitive, problem solving and critical thinking skills among the students. The strategy also helps the students to work in group and develop a democratic attitude and to judge a problem based on consensus and concluded that PBL should be implemented to achieve critical thinking, scientific thinking, leadership qualities and managerial skills among students.

Additionally, L'Ecuyer et al., (2015) who conducted a study about "The use of PBL in an interprofessional education course for health care professional students" and indicated that PBL is an effective method for teaching interprofessional collaboration skills to nursing students by allowing them to practice needed skills with an interprofessional team in a seminar setting.

Also, Klunklin et al., (2011) who conducted a study about "Thai nursing students' adaption to problem-based learning: A qualitative study" and concluded that PBL could be attributed to increased ability to problem solve, and satisfaction with creativity in learning, group work, and leadership development. On the other hand, O'Donoghue et al., (2011) found no proven evidence that it improved practice performance, or enhanced student satisfaction. They found a weak effect for enhanced approaches to learning.

\section{Part V. Attitude toward PBL among studied PBL group at pre and follow up phases}

This part answers the third study objective which was identifying intern-nurses' attitude toward problem-based learning.

Concerning intern-nurses' attitude toward PBL, the results of the present study showed that the majority of intern-nurses had positive attitude toward PBL at follow up 
Enhancing Managerial Skills for Intern-Nurses by Using Problem-Based Learning Strategy

phase compared to pre-program. Also, there was highly statistical significant difference between pre-program and follow up phase regarding attitude of intern-nurses toward PBL.

This might be due to PBL is interesting, enhance knowledge and skills, encourage intern-nurses to be active, provide supportive environment and learning experiences more than other strategies, stimulate the ability to think, encourage intern-nurses to share ideas and different opinions, different intern-nurses have different strength that help in solution of problems, students are individually accountable or responsible for their work and the students working together in small group to accomplish a common task. Also, Positive learning attitudes includes the followings: improved depth of learning in sessions, consistency of learning, repetition and group discussion in class, increased motivation to search the litterateurs and use references, opportunity for interaction with faculty members and peers.

On the same line, Sangma et al., (2019) who conducted a study entitled "Perspectives on problem-based learning " and found that overall students had negative perception before PBL introduction. After PBL introduction majority of students showed a strong positive response of all items and there was significant increase in the score.

Also, Arman, (2018) who conducted study about "Students' attitudes toward problem based learning - Analog electronic course in the electrical engineering programs in PPU case study" and indicated positive attitudes of students toward this new approach of learning. In addition, Yadav et al., (2018) found that student's attitude toward PBL was positive. They highly appreciated the roles of a tutor as facilitators and motivators for proper activities in PBL session. Moreover, Bruce et al., (2018); AbdRabou, (2017) and Abd El-Hay and Abd-Allah, (2015) found that the majority of nursing students felt that PBL was a stimulating and useful learning strategy, with most students rating their attitude positively.

Besides, Jindal et al., (2016); Agbeh, (2015); Othman and Shalaby, (2014); Bell, (2012) and Hamza, (2012) showed that students' attitudes toward PBL were positive which can improve their abilities in gaining reasoning skills. Also, Surif et al., (2013) who conducted a study entitled "Implementation of problem based learning in higher education institutions and its impact on students' learning" and showed that students had positive perceptions towards the implementation of PBL in their learning process.

In addition, Sharma and Sachdeva, (2017) found out the satisfaction of students with this learning method and the students' interest and ability for improvement after this teaching method. As well, Wakode et al., (2016) who conducted a study entitled "Perception on problem based learning sessions amongst first year medical graduates of central India" and found that student's response towards PBL was favorable. On the other hand, this is in contrast with findings of Al-Naggar and Bobryshev, (2012) who conducted a study entitled "Acceptance of problem based learning among medical students" and found that the acceptance of PBL among medical students was quite low.

\section{Part VI. Evaluation of studied PBL group thorough program phases}

This part answers the fifth study objective which was evaluating the 
Asmaa Khaled Abd El-Aziz, Gehan Mohamed Ahmed Mostafa, Ebtesam Saeed Ahmed

effectiveness of the designed program on intern-nurses' managerial knowledge and skills.

Concerning tutor evaluation of the intern-nurses, the results of the current study showed that there was highly statistical significant difference improvement in performance of intern-nurses as evaluated by tutor. The majority of intern-nurses had good performance at immediate post and follow up phase respectively. This might be due to PBL provides supportive and challenged environment that motivate and enhance students to learn and gain skills.

In the same line, this finding was congruent with Othman and Shalaby, (2014) who demonstrated that during tutorial evaluation of student's performance' throughout PBL sessions, the students developed good level of performance regarding utilization of learning resources, critical appraisal skills, group work activities and attitudes and communication skills.

\section{Also, Abd El-Raouf and Ahmed,} (2011) who found that tutorial evaluation revealed that students were able to correlate theoretical knowledge to solve the assigned scenario. About one third of students showed a good level in self-learning skills such as stating their learning goals and managing their learning limitations. Also, revealed that students highly rated their performance in PBL sessions, as more than half of students up to more than two thirds rated themselves as excellent in the majority of PBL skills.

Concerning intern-nurses' selfevaluation the results showed that at preprogram phase more than half of intern-nurses had poor performance, however the majority of them had good performance at immediate post and follow up phases as reported by intern-nurses themselves. This might be due to the positive effect of PBL on students.

On the same line, Soliman et al., (2017) who conducted a study about "Postgraduate nursing students' acquire skills upon applying problem based learning" and revealed that the postgraduate students showed very good level of performance in relation to interpret the information and attainment of the team's learning objectives. Moreover, the results showed improvement of learner abilities in self-directed learning, critical thinking, independent study, group interaction, reasoning skills, and active participation. Moreover, post graduate students utilized library and internet, they gained teamwork and collaboration skills.

Furthermore, Abd El-Hay and AbdAllah, (2015) who revealed that PBL improve students' ability to make self-evaluation and found that nursing students' self-evaluation about their experience with PBL strategy were positive. Also participation, communication and decision making skills were developed after implementation of problem based learning strategy.

Besides, Larin et al., (2010) who conducted a study entitled "Students' perspectives on problem-based learning in a transitional doctorate of physical therapy program" and demonstrated that students described improved skills in professional behavior, communication, critical inquiry, clinical decision making, reflection, and evidence-based practice. Students wrote in their reflective journals that they had learned how to access, evaluate and apply appropriate information to practice, communicate effectively with colleagues and clients, and reflect on their actions and decisions. 
Enhancing Managerial Skills for Intern-Nurses by Using Problem-Based Learning Strategy

Part VII. Correlations among studied variables at different study phases

The result of present study revealed that there was highly statistical significant positive correlation between total managerial skills knowledge and total managerial skills' practice at both groups. This means that when managerial skills knowledge increase, managerial skills' practice will increase also. From the researcher point of view, this might be due to that having the knowledge serves as a powerful route and the basis to help the intern-nurses to practice.

In the same line, this finding was congruent with Mohamed, (2013) who conducted a study entitled "Intervention program for nurse interns about managerial skills" and found that there was positive correlation with statistically significant relation between managerial skills knowledge and practice throughout program phases.

Also, the result of present study revealed that there was a highly statistical significant positive correlation between internnurses' managerial skills knowledge, practice and total attitude, opinionnaire, total performance and self-evaluation scores thorough program phases. This might be due to increasing knowledge and practice related to managerial skills after PBL will have positive effect on the attitude and opinionnaire toward PBL. Also, this will have effect on the intern-nurses' performance.

This result was supported with Hande et al., (2015) who found positive correlation between acquisition of knowledge and generic skills, that between generic skills and attitude and that between acquisition of knowledge and attitude. There was a significant positive correlation between the scores of the three domains for all batches of students.

\section{Conclusion}

In the light of the foregoing present study results, it can be concluded that, the majority of experimental intern-nurses preferred PBL over TBL at immediate post and follow up phases as a strategy of learning. Moreover, the majority of intern-nurses had a positive attitude toward PBL at follow up phase compared to pre-program. Also, there was statistical significant improvement regarding internnurses' managerial skills knowledge and practice in experimental group (PBL group) than control group (TBL). Additionally, PBL group reported high satisfactory level of managerial skills' knowledge and practice than TBL group at immediate post and follow up phase. There was highly statistical significant difference improvement in the performance of intern-nurses as evaluated by tutor and by intern-nurses themselves. Furthermore, there was highly statistical significant positive correlation between total managerial skills' knowledge and total managerial skills' practice at both groups. These findings support research hypotheses.

\section{Recommendations}

In the light of the findings obtained from the present study, the following recommendations are suggested:

$\checkmark$ Nursing educational administrators should assure and providing supportive and empowering learning environment as prerequisites to PBL implementation.

$\checkmark$ PBL strategy should be adopted by Faculty of Nursing Benha University for better skills and learning outcome, to allow students' interactions and encourage higher thinking level. 
Increase awareness among faculty members by providing a series of workshops and training related to use of the PBL to be used more effectively.

$\checkmark$ Nursing internship orientation program should include topics related to essential managerial skills needed to be applied in internship year that foster and support intern-nurses in the clinical area.

$\checkmark$ Hospital should support empowerment, enabling intern-nurses to be responsible and accountable for their professional practice under supervision to become confident in their clinical and management skills.

$\checkmark$ As a nursing student, it is important to get involved in and make sure internship reflects what you have learned.

\section{Future researches are suggested}

$\checkmark$ Conduct a study to evaluate the impact of PBL on nursing students' transition to clinical practice and lifelong learning.

$\checkmark$ Reapplication of the study on larger probability sample at different nursing faculties and universities in an effort to more fully understand the processes involved in planning and implementing PBL program.

$\checkmark$ Conduct a study to assess factors affecting the nursing students' learning motivation in the PBL as learning method.

$\checkmark$ Conduct a study to examine the effect of pure PBL, hybrid PBL and lecture on the nursing students' achievement and performance.

\section{References}

Abd El-Hay,S. and Abd-Allah,S. (2015): Effect of problem-based learning strategy on development of problem solving skills among undergraduate nursing students, IOSR Journal of Nursing and Health Science, 4(3): 1-13.

Abd-Elmonem,A., Eid,N. and Rashad,R. (2019): Enhancing nurses toward internal marketing and its effect on patients' outcome at Benha University Hospital, IOSR Journal of Nursing and Health Science, 8(1 Ser. VII.): $28-40$.

Abd El-Raouf,S. and Ahmed,A. (2011): Nursing students' experiences with problem based learning: a teaching strategy applied in community health course, Med. J. Cairo Univ., 79(1): 323-333.

Abd Rahman,M., Azmi,M., Wahab,Z., Abdullah,A. and Azmi,N. (2016): The impacts of problem-based learning approach in enhancing critical thinking skills to teaching literature, International Journal of Applied Linguistics \& English Literature, 5(6): 249-258.

AbdRabou,F. (2017): Problem based learning for improving group dynamic: nursing students self report and perception, IOSR Journal of Nursing and Health Science, 6 (3 Ver. VI): 27-34.

Adiga,U. and Adiga,S. (2015): Problem based learning, International Journal of Current Research, 7(6): 17181-17187.

Agbeh,A. (2015): Problem-based learning in a hospitality and tourism administration course, doctorate thesis, The Graduate School, Southern Illinois University of Carbondale, pp.23-44,173.

Ahamad,S., Li,H-C., Shahrill,M. and Prahmana,R. (2017): Implementation of problem-based learning in geometry lessons, Journal of Physics: Conf. Series, 943 (1):1-15.

Ahmed,I. (2013): Study the effectiveness of implementing team building strategies program for nursing-interns by using teambased learning at Benha University Hospital, 
Enhancing Managerial Skills for Intern-Nurses by Using Problem-Based Learning Strategy doctorate thesis, faculty of Nursing, Benha University, PP 59-60.

Ahmed,M. (2012): Time management challenges among jordanian nurse managers, Life Science Journal, 9(3): 2464- 2469.

Alajmi,N. (2014): Factors that influence performance in a problem-based learning tutorial, doctorate thesis, Faculty of Health Sciences and Medicine, Bond University,pp 14-18, 71-80.

Alarcon,M. and Prezotto,K. (2016): Evaluation of educational strategy, grounded on problem-based learning on nursing undergraduate, Rev Rene, 17(2):242-249.

Allareddy,V., Havens,A., Howell,T. and Karimbux,N. (2011): Evaluation of a new assessment tool in problem-based learning tutorials in dental education, Journal of Dental Education, 75(5): 665-671.

Almasoudi,B.(2012): Problem-based learning as a teaching method versus lecture-based teaching in respiratory therapy education, master thesis, Byrdine F. Lewis School of Nursing and Health Professions, Georgia State University, pp 1-4, Available at http://scholarworks.gsu.edu/rt_theses/13,

Retrived on 28/12/2018 at 12:30 P.M.

Al-Naggar,R. and Bobryshev,Y. ( 2012): Acceptance of problem based learning among medical students, Journal of Community Medicine \& Health Education, 2(5):1-6.

Alper,A. (2008): Attitudes toward problem based learning in a new Turkish medicine curriculum, World Applied Sciences Journal, 4 (6): 830-836.

Alrahlah,A. (2016): How effective the problem-based learning (PBL) in dental education. a critical review, The Saudi Dental Journal, 28(4): 155-161.

AlThiga,H., Mohidin,S., Park,Y. and Tekian,A. (2017): Preparing for practice:

nursing intern and faculty perceptions on clinical experiences, Med Teach, 39(sup1): S55-S62.

Ansari,M., Abd Rahman,S., Badgujar,V., Sami,F. and Abdullah,M. (2015): Problem based learning (PBL): a novel and effective tool of teaching and learning, Indian Journal of Pharmaceutical Education and Research, 49 (4): 258-265.

Arman,A. (2018): Students' attitudes toward problem based learning-analog electronic course in the electrical engineering programs in PPU case study, Journal of eLearning and Higher Education, 2018 (2018): 1-8.

Assumpção,W., Stettiner,C., Filho,M., Moia,R. and Ferreira,V. (2016): Using problem-based learning in entrepreneurship education, Australian Journal of Basic and Applied Sciences, 10(10): 237-242.

Augusthy,V. (2016): A comparative study of the learning outcomes and students' satisfaction from problem-based learning and lecture-based learning in obstetrics and gynaecology, International Journal of Reproduction, Contraception, Obstetrics and Gynecology, 5(5): 1368-1374.

Bakr,M., Sherif,N., Eid,N. and ELshal,S. (2013): Factors influencing decision making and its effect on intern students clinical performance, World Applied Programming, 3(2): 75-84.

Banu,A., Khan,K. and Rajkumar,J. (2014): Problem based learning in medical educationa review, Journal of Educational Research \& Medical Teacher, 2(2):1-5.

Bell,J. (2012): Introducing problem-based learning as a learning strategy for masters students, Practitioner Research in Higher Education, 6(1):3-11. 
Asmaa Khaled Abd El-Aziz, Gehan Mohamed Ahmed Mostafa, Ebtesam Saeed Ahmed

Bolfíková,E., Hrehová,D. and Frenová,J. (2010): Manager's decision-making in organizations-empirical analysis of bureaucratic vs. learning approach, Journal of Economics and Business, 28(1): 135-163.

Bruce,J., Lack,M., Bomvana,N. and Qamata-Mtshali,N. (2018): Problem-based learning: nursing students' attitude, selfreported competence, tutorial performance and self-directed learning readiness, Journal of Nursing Education and Practice, 8(10): 1119.

Bulog,I., Dulčić, Ž. and Sekulović,A. (2017): Managerial skills in hotel industryevidence from Croatia, Dubrovnik International Economic Meeting, 3(1): 419432.

Burgess,A., Roberts,C., Ayton,T. and Mellis,C. (2018): Implementation of modified team-based learning within a problem based learning medical curriculum: a focus group study, BMC Medical Education, 18(74): 1-7.

Chia, L. and Chin, C. (2008): Problem based learning tools, The Science Teacher, 75(8): 44-49.

Dahlkemper,T. (2018): Nursing leadership, management, and professional practice for the LPN/LVN, 6th ed., F.A. Davis company, United states of America, Available at https://books.google.com.eg/books?,

Retrieved on 15/1/2018 at 4:00 A.m.

De Witte,K. and Rogge,N. (2012): Problembased learning in secondary education: evaluation by a randomized experiment, Education Economics, 24(1): 1-22.

Deep,S., Salleh,B. and Othman,H. (2016): Exploring the role of problem-based learning in developing conflict resolving and other soft skills-a quasi-experimental study, International Review of Management and Marketing, 6(4): 738-748.
deNevers,D.(2014):Interpersonal intelligence and

problem-based learning, published master of education program thesis, Dordt College Sioux Center, Iowa, pp 1-7, Available at http://digitalcollections.dordt.edu/med_theses, Retrived on 21/12/2018 at 10:30 P.M.

Dhami,D., Rathore,R., Bhargava,V., Neupane,G., Singh,R., Singh,A. and Shah,G. (2017): Student's attitude toward lbl versus pbl in pharmacology at Ngmc, Banke, Nepal, International Journal of ResearchGranthaalayah, 5(9): 244-249.

Ding,X., Zhao,L., Chu,H., Tong,N., Ni,C., Hu,Z., Zhang,Z.

and Wang,M. (2014): Assessing the effectiveness of problem-based learning of preventive medicine education in China, Scientific Reports, 4 (5126): 1-5.

Dissanayaka,T., Marambe,K. and Liyanage,E. (2012): Physiotherapy students' perception on problem based learning, Sri Lanka Journal of Bio-Medical Informatics,3(3):75-81.

El-Sayed,S., Metwally,F. and Abdeen,M. (2013): Effect of peer teaching on the performance of undergraduate nursing students enrolled in nursing administration course, Journal of Nursing Education and Practice, 3(9): 156166.

EL-Shaer,A. and Gaber,H. (2014): Impact of problem-based learning on students`critical thinking dispositions, knowledge acquisition and retention, Journal of Education and Practice, 5(14):74-85.

Emerald,N., Aung,P., Han,T., Yee,K., Myint,M., Soe,T. and Oo,S. (2013): Students' perception of problem-based learning conducted in phase1 medical program, UCSI University, Malaysia, South 
Enhancing Managerial Skills for Intern-Nurses by Using Problem-Based Learning Strategy

East Asian Journal of Medical Education, 7 (2): 45-48.

\section{Esfandnia,F., Khezeli,M., Bayat,R.,} Ojaghi,S. and EsfandniaA.(2016): Relationship between the managerial skills with the effectiveness of managers, Acta Medica Mediterranea, 32(2): 987-990.

Faisal,R., Rehman,K., Bahadur,S. and Shinwari ,L. (2016): Problem-based learning in comparison with lecture-based learning among medical students, Journal of the Pakistan Medical Association, 66(6):650-653.

Fatima,S., Ghias,K., Jabeen,K. and Sabzwari ,S. (2019): Enhancing cognitive engagement of pre-clinical undergraduate medical students via video cases and interactive quizzes in problem-based learning, Cureus, 11(1): e3832.(1-16).

Frambach,D. (2012): Rethinking the globalization of problem-based learning: how culture challenges selfdirected learning, Medical Education, 46(8):738-747.

Gassas,R., Mahran,S. and Banjar,H. (2017): Nurse managers' attitude and competency towards delegation in Jeddah city, American Journal of Nursing Science, 6(2): 72-79.

Ghimire,S. and Bhandary,S. (2014): Students' perception and preference of problem based learning during introductory course of a Nepalese medical school, Journal of Patan Academy of Health Sciences, 1(1):64-68.

Gönc,V., Lorber,M. and Nerat,J. (2017): Experience of problem- based learning for raising quality of nursing study, teaching and learning in nursing, Dr. Gregor Stiglic (Ed.), InTech, DOI: 10.5772/67427.pp 17-25, Available from: https://mts.intechopen.com/books/teachingand-learning-in-nursing/experience-ofproblem-based-learning-for-raising-quality- of-nursing-study, Retrived on 28/3/2018 at 10:34 P.M

Hamza,R. (2012): Effect of problem based learning and lecture based learning on nursing students at Oman nursing institute, Kufa Journal For Nursing Sciences, 2(2): 1-12.

Hande,S., Mohammed,C. and Komattil,R. (2015): Acquisition of knowledge, generic skills and attitudes through problem-based learning: student perspectives in a hybrid curriculum, Journal of Taibah University Medical Sciences, 10(1): 21-25.

Heaviside,H., Manley,A. and Hudson,J. (2018): Bridging the gap between education and employment: a case study of problembased learning implementation in postgraduate sport and exercise psychology, Higher Education Pedagogies, 3(1): 463-477.

Hung, C-H. and Lin, C-Y. (2015): Using concept mapping to evaluate knowledge structure in problem-based learning, BMC Medical Education, 15 (212): 1-9.

Ibrahim,N., Banjar,S., Al-Ghamdi,A., AlDarmasi,M., Khoja,A., Turkistani,J., Arif,R., Al-Sebyani,A., Musawa,A. and Basfar,W. (2014): Medical students' preference of problem-based learning or traditional lectures in King Abdulaziz University, Jeddah, Saudi Arabia, Ann Saudi Med, 32(4):128-133.

Imran,M., Shamsi,M., Singh,A., Goel,S., Sharma,P. and Panesar,S. (2015): Problembased learning versus lecture-based learning in pharmacology in a junior doctor teaching program: a crossover study from northern India, International Journal of Research in Medical Sciences, 3(11):32963299.

Jindal,M., Mahajan,H., Srivastav,S. and Baro,G. (2016): Pros and cons of problembased learning in medical education: students' viewpoint, NJIRM, 7(4): 77-81. 
Asmaa Khaled Abd El-Aziz, Gehan Mohamed Ahmed Mostafa, Ebtesam Saeed Ahmed

Kartikeyan,S. and Malgaonkar,A. (2018):

Comparison of cognitive domain scores after lecture-based and problem-based learning in community medicine, International Journal of Community Medicine and Public Health, 5(10):4527-4531.

Kelly,P. (2010): Essentials of nursing leadership \& management, 2nd ed., Delmar, Cengage Learning, the United States of America,pp.104, 59-162, 188-190.

Keshk,L., Abd El-Azim,S. and Qalawa,S. (2016): Quality of problem based learning scenarios at college of nursing in egypt and ksa: comparative study, American Journal of Educational Research, 4(9): 701-710.

Khoshnevisasl,P.,

Sadeghzadeh,M., Mazloomzadeh,S., Feshareki,R. and Ahmadiafshar,A. (2014): Comparison of problem-based learning with lecture-based learning, Iran Red Crescent Med J., 16(5): e5186: 1-4.

Kim,Y. (2019): Observational application comparing problem-based learning with the conventional teaching method for clinical acupuncture education, Evidence-Based Complementary and Alternative Medicine, 2019 (9): 1-6.

Klunklin,A., Subpaiboongid,P., Keitlertnapha,P., Viseskul,N. and Turale,S. (2011): Thai nursing students' adaption to problem-based learning: a qualitative study, Nurse Education in Practice, 11, 370-374. http://dx.doi.org/10.1016/j.nepr.2011.03.011.

Larin,H., Buccieri,K. and Wessel,J. (2010): Students' perspectives on problembased learning in a transitional doctorate of physical therapy program, Journal of the Scholarship of Teaching and Learning, 10(3): 128-144.

L'Ecuyer,K., Pole,D. and Leander,S. (2015): The use of PBL in an interprofessional education course for health care professional students, Interdisciplinary Journal of Problem-Based Learning, 9(1): 918.

Looi,H. and Seyal,A. (2014): Problem-based learning: an analysis of its application to the teaching of programming, International Proceedings of Economics, 70(14):68-75.

Mahdy,A. and Mahfouz,H. (2016): Evaluate the effectiveness of nurses' professional competence approach on their productivity in medical-surgical units, Egyptian Journal of Health Care, 7(3): 271291.

Mahmoud,H. (2017): The effect of cooperative learning strategy on undergraduate nursing students enrolled in nursing administration course, International Journal of Learning and Development, 7(1): 23-40.

Majagi,S. and Torgal,S. (2015): Introduction of designing of problem in problem based learning in pharmacology for under-graduates, American Journal of Pharmacology and Pharmacotherapeutics, 2(4): 95-111.

Makhlof,E. and El-Saman,S. (2017): Internship nurses' satisfaction with clinical learning environment in intensive care unit, IOSR Journal of Nursing and Health Science, 6(2 Ver. V): 112-119.

McMahon, M., Luca, J. and John, C. (2007): A self assessment tool to help learners develop teamwork skills, Proceedings of World Conference on Educational Multimedia, Hypermedia and Telecommunications, pp. 2913-2920, Available at http://ro.ecu.edu.au/ecuworks/1609, Retrived on 19/1/2019 at 1:36 A.M.

Meo,S. (2013): Evaluating learning among undergraduate medical students in schools 
Enhancing Managerial Skills for Intern-Nurses by Using Problem-Based Learning Strategy

with traditional and problem-based curricula, Adv Physiol Educ., 37(3): 249-253.

Mohamad, H. (2017): The impact of problem-based learning on students' competencies in technical vocational education and training, Aalborg Universitetsforlag, Published Doctorate thesis-serien for Det Tekniske Fakultet for IT og Design, Aalborg Universitet, pp 27-29, 4245 , Available at http://vbn.aau.dk/files/267747935/PHD, Retrived on 10/7/2018 at 4:00 P.M .

Mohamed,A. (2013): Intervention program for nurse interns about managerial skills, doctorate theses, Faculty of Nursing, Zagazig University, Pp:91-96.

Mohan,L., Shenoy,S., Eesha, B.,Anoopkishore,A., Bairy,K. and Patil,N. (2014): Students' attitude toward didactic lecture versus problem-based learning in pharmacology: a questionnaire based study, International Journal of Basic \& Clinical Pharmacology, 3(4): 619- 622.

Molande,E., Mtemang'ombe,D. and Chikasanda,V. (2017): Effectiveness of problem-based learning for technical teacher training in woodwork at a Malawi college, International Journal of Vocational and Technical

Education, 9(5): 40-48.

Morsy,S. (2010): Relationship between time management skills and effective delegation among the head nurses at Assuit University Hospitals, Al-Azhar Assiut Medical Journal (AAMJ), 8(3):27-43.

O'Donoghue,G., McMahon,S., Doody,C. and Cusack,T. (2011): Problem-based learning in professional entry-level therapy education: a review of controlled evaluation studies, Interdisciplinary Journal of Problembased Learning, 5(1): 54-73.
Olmstead,J. (2010): Effective time management skills \& practices: selfassessment questionnaire, pp 1-7, Available at https://www.olmsteadassoc.com/wpcontent/u ploads/2017/01/Effective-Time-ManagementSkills-Self-Assessment-Questionnaire.pdf, Retrived on 31/12/2017 at 1:30 A.m.

Orji,C. and Ogbuanya,T. (2018): Assessing the effectiveness of problem-based and lecture-based learning environments on students' achievements in electronic works, International Journal of Electrical Engineering Education, 55(4): 334-353.

Othman,H., Salleh,B. and Sulaiman,A. (2013): 5 Ladders of active learning: an innovative learning steps in PBL process, The 4th International Research Symposium on Problem-Based Learning (IRSPBL) 2013, pp 245-253.

Othman,S. and Shalaby,S. (2014): Students' perception and acceptance of problem-based learning approach in critical care nursing practice, Scientific Cooperations International Workshops on Medical Topics, AnkaraTURKEY, 7-8 June 2014: 52-67.

Padmanabha,T., Manu,G., Madhav,K., Chandrakantha,T. and Neha, K. (2016): Student's perception towards learning medical sciences: problem based learning versus lecture based learning methods, International Journal of Basic \& Clinical Pharmacology, 5(2): 411-415.

Parsons,L. (2015): Leadership and management for every nurse, 2nd ed., Nurses Continuing Education-CE Express Home Study, Available at http://www.ceexpress.com/Portals/11/pdfs/N1664.pdf, Retrieved on 7/1/2018 at 3:27 P.M.

Pourshanazari,A., Roohbakhsh,A., Khazaei,M. and Tajadini,H. (2013): Comparing the long-term retention of a physiology course for medical students with 
the traditional and problem-based learning, Adv Health Sci Educ,18(1): 91-97.

Pryby,F. (2016): Improving the charge nurse's leadership role: a collaborative learning forum, doctorate theses, College of Health Sciences, Walden University, Pp.1226.

Qalawa,S. and Keshk,L. (2014): Impact of problem based learning on acquiring 21 century skills among nursing students (comparative study), International Journal of Advanced Research, 2(7): 770-783.

Rakhudu,M., Davhana-Maselesele,M. and Useh,U. (2017): A model of collaboration in implementing problem-based learning $(\mathrm{Pbl})$ in nursing education in South Africa, Curationis,40(1): e1-e10.

Rashid,R. (2011): A perspective evaluation of problem-based learning in Esl classroom in the Malaysian higher school certificate program, i-manager's Journal on English Language Teaching, 1(4): 25-32.

Rubenfeld,M. and Scheffer,B. (2010): Critical thinking tactics for nurses: achieving the IOM competencies, 2nd ed., jones and Bartlett publishers, boston, toronto, London, pp 25-44, 259-285.

Sangma,M., Lyngdoh,J., Barooah,R., Visi,V., Longkumer,C. and Biaklhupuii,C. (2019): Perspectives on problem-based learning (PBL), IOSR Journal of Dental and Medical Sciences, 18(4): 08-11.

Sathya,P. and Reddemma,K. (2017): Development of problem based learning training module in nursing education, International Journal of Research in Medical Sciences, 5(5):1986-1990.

Sayyah,M., Shirbandi,K., Saki-Malehi,A. and Rahim,F. (2017): Use of a problembased learning teaching model for undergraduate medical and nursing education: a systematic review and meta-analysis,
Advances in Medical Education and Practice, 8(1): 691-700.

Sharma,P. and Sachdeva,K. (2017): Students' perception to introduction of problem-based learning modules in anatomy, Curr trends Diagn Treat, 1(1): 42-45.

Sharma,R. (2015): Effect of problem based learning on nursing students' clinical decision making and learning satisfaction, International Journal of Science and Research, 4(7): 163-165.

Shin,I. and Kim,J. (2013): The effect of problem-based learning in nursing education: a meta-analysis, Advances in Health Science Education,18(5): 1103-1120.

Soliman,S., Abd El -Mouty,S. and Salem,N. (2017): Postgraduate nursing students' acquire skills upon applying problem based learning, IOSR Journal of Nursing and Health Science, 6 (2 Ver. VI): 5-11.

Strobel,J. and van Barneveld, A. (2009): When is PBL more effective? A metasynthesis of meta-analyses comparing PBL to conventional classrooms, The Interdisciplinary Journal of Problem-based Learning, 3 (1): 44-58.

Strom,P. and Strom,R. (2011): Teamwork skills assessment for cooperative learning, Educational Research and Evaluation, 17(4): 233-251.

Sullivan,E. and Decker,P. (2009): Effective leadership and management in nursing, 7th ed., pearson education international, united states of America, Pp 135-142, 164-71.

Surif,J., Ibrahim,N. and Mokhtar,M. (2013): Implementation of problem based learning in higher education institutions and its impact on students' learning, The 4th International Research Symposium on Problem-Based Learning, Pp 66-73. 
Enhancing Managerial Skills for Intern-Nurses by Using Problem-Based Learning Strategy

Tayyeb,R. (2013): Effectiveness of problem based learning as an instructional tool for acquisition of content knowledge and promotion of critical thinking among medical students, Journal of the College of Physicians and Surgeons Pakistan, 23 (1): 42-46.

Tian,J., Liu,A., Yang,K. and Shen,X. (2013): the effectiveness of problem-based learning on evidence based medicine: a double-blind randomized trial, Indian Journal of Pharmaceutical Education and Research, 47 (3): 14-18.

Valle,R., Petra,L, Martínez-Gonzáez,A., Rojas-Ramirez,J., Morales-Lopez,S. and Piña-Garza,B. (1999): Assessment of student performance in problem-based learning tutorial sessions, Medical Education, 33(11):818-822.

Wakode,S., Wakode,N., Khobragde,R., Tambe,M., Tajne,V. and Mishra,N. (2016): Perception on problem based learning sessions amongst first year medical graduates of central India, Scholars Journal of Applied Medical Sciences (SJAMS), 4(7D):25722574.

Wang,J., Xu,Y., Liu,X., Xiong,W., Xie,J. and Zhao,J. (2016): Assessing the effectiveness of problem-based learning in physical diagnostics education in China: a meta-analysis, Scientific Reports, 6, 36279, doi: 10.1038/srep36279: 1-7.

Whetten,D. and Cameron,K. (2011): Developing management skills, 8th ed., Pearson Education, Inc., publishing as Prentice Hall, The United States of America, pp.10-11, 373- 450.

Wosinski,J., Belcher,A., D“urrenberger,Y., Allin,A., Stormacq,C. and Gerson,L. (2018): Facilitating problem-based learning among undergraduate nursing students: A qualitative systematic review, Nurse Education Today, 60(1): 67-74.
Wright,D., Wickham,J. and Sach,T. (2014): Problem-based learning: An exploration of student opinions on its educational role in one UK pharmacy undergraduate curriculum, International Journal of Pharmacy Practice, 22(3): 223-230.

Yadav,R., Piryani,R., Deo,G., shah,D., Yadav,L. and Islam,M. (2018): Attitude and perception of undergraduate medical students toward the problem-based learning in chitwan medical college, Nepal, Advances in Medical Education and Practice, 9(2018):317-322.

Yang,J. and Yang,B. (2013): Nursing students' experiences with facilitator in problem-based learning class, Asian Nursing Research,7(4):198-204.

Yaqinuddin,A. (2013): Problem-based learning as an instructional method, Journal of the College of Physicians and Surgeons Pakistan, 23(1): 83-85.

Yew,E. and Goh,K. (2016): Problem-based learning: an overview of its process and impact on learning, Health Professions Education, 2(2): 75-79.

Yi,Q. (2016): International nursing students' experiences of problem-based learning in a university of applied sciences, master thesis, Faculty of Health Sciences, University of Eastern Finland, PP 55-59., Available at https://epublications.uef.fi/pub/urn_nbn_fi_ue f-20161279/urn_nbn_fi_uef-20161279.pdf, Retrived on $14 / 12 / 2019$ at 10:58 P.M.

Alenezi, N., Elboraei ,Y., and Alruwaili, A. (2017). Ear diseases and factors associated with ear infections among the elderly attending hospital in Arar city, Northern Saudi Arabia. J of Electronic Physician. 9(9): 5304-5309. 


\section{تعزيز المهارات الإدارية لتمريض الإمتياز بإستخدام إستراتيجية التعلم القائم على المشكلة} أسماء خالد عبدالعزيز زكى, جيهان محمد أحمد مصطفى, إبتسام سعيد أحمد عبدالرحمن

تم تطبيـق التعلم القائم على حل المشكلات في جميع أنحـاء العـالم كطريقة تعليميـة مبتكرة في تعليم التمريض ، وقد تبنت بعض الجامعات المصرية التعلم القائم على حل المشكلات كطريقة تعليمية تهدف إلي تَتجيع طُلاب التمريض ليس فقط على اكتساب المحتوى، ولكن لاكتثـاف المعلومـات وتنقيتها ودَمجها من أجل هُمارسة مهار ات التفكير العليا. لذا هَدفت هذه الدر اسه إلي تَعزيز المهار ات الإداريـة لتمريض الإمتيـاز بإستخدام استر اتيجية التعلم القائم على المشكلة في مستشفى جامعة بنها, وقد انتَهَجت الدراسة المنهج شبه التجريبي لتحقيق الهذف منها و إثنَملت عينـة الدر اسـة على 124 من تمريض الإمتياز : منهم 54 فرد تم وضعهم فيى المجموعـة التجريبية/الدر اســة (الـتعلم القـائم على المشـكلة), و70 فـرد تـم وضـعهم فـى المجموعــة الضــابطه (الطريقـة التقليدية/المحاضـرة). حيث أظهرت النتائج بـان هنـاك تحسن كبيـر ذات دلالـة إحصـائية بـين المجمـوعتين مـع متوسط درجات أعلى في المجموعة التجريبية عن المجمو عـة الضـابطه بعد تنفيذ البرنـامج مباشرة مع وجود علاقة ذات دلالة احصـائية بين معلومـات المهار ات الإداريـة الكلية وممارسـة المهار ات الإداريـة الكلية في كلا المجمو عتين. وأوصت الدر اسة إلى تَنبى استر اتيجية التعلم القائم على المشكلة من قبل كلية التصريض جامعة بنها لتحسين المهار ات ونتائج التعلم ، و السماح بتفاعل الطلاب وتشجيع مستوى التفكير العالي لديهم 